TRANSACTIONS OF THE

AMERICAN MATHEMATICAL SOCIETY

Volume 366, Number 2, February 2014, Pages 563-589

S 0002-9947(2013)05726-1

Article electronically published on August 14, 2013

\title{
KÄHLER-RICCI FLOW ON PROJECTIVE BUNDLES OVER KÄHLER-EINSTEIN MANIFOLDS
}

\author{
FREDERICK TSZ-HO FONG
}

\begin{abstract}
We study the Kähler-Ricci flow on a class of projective bundles $\mathbb{P}\left(\mathcal{O}_{\Sigma} \oplus L\right)$ over the compact Kähler-Einstein manifold $\Sigma^{n}$. Assuming the initial Kähler metric $\omega_{0}$ admits a $U(1)$-invariant momentum profile, we give a criterion, characterized by the triple $\left(\Sigma, L,\left[\omega_{0}\right]\right)$, under which the $\mathbb{P}^{1}$-fiber collapses along the Kähler-Ricci flow and the projective bundle converges to $\Sigma$ in the Gromov-Hausdorff sense. Furthermore, the Kähler-Ricci flow must have Type I singularity and is of $\left(\mathbb{C}^{n} \times \mathbb{P}^{1}\right)$-type. This generalizes and extends part of Song-Weinkove's work on Hirzebruch surfaces.
\end{abstract}

\section{INTRODUCTION}

The Ricci flow was introduced by Hamilton in his seminal paper H1] in 1982, proving the existence of a constant sectional curvature metric on any closed 3manifold with positive Ricci curvature. Since then, the Ricci flow has been making breakthroughs in settling several long-standing conjectures. Just to name a few, based on a program proposed by Hamilton, a complete proof of the Poincaré conjecture was given by Perelman [P1, [P2, [P3] around 2003. See also [CZ, KL, MT]. Furthermore, the Differentiable Sphere Theorem was proved by Brendle-Schoen [BS] in 2007, giving an affirmative answer to a conjecture about differential structures of quarter-pinched manifolds proposed by Berger and Klingenberg in the 1960s. In the realm of Kähler geometry, the Kähler-Ricci flow was introduced by Cao in Cao1, which proves the smooth convergence towards the unique Kähler-Einstein metric in the cases $c_{1}<0$ and $c_{1}=0$.

There has been much interest in understanding the limit behavior and singularity formation of the Ricci flow in both Riemannian and Kähler settings. Hamilton introduced in [H3] a method of studying singularity formation of the Ricci flow by considering the Cheeger-Gromov limit of a sequence of rescaled dilated metrics. The singularity model that was obtained, which is often an ancient or eternal solution, captures the geometry of the singularity formation near the blow-up time of the flow. For closed 3-manifolds, the study of ancient $\kappa$-solutions formed by the dilated sequence limit in Hamilton-Perelman's works (e.g. [H3, P1]) leads to a solid understanding of singularity formation of closed 3-manifolds.

Another way of interpreting singularity formation is by the Gromov-Hausdorff limit, regarding the manifold as a metric space. This notion was recently employed

Received by the editors April 13, 2011 and, in revised form, September 30, 2011 and October $12,2011$.

2010 Mathematics Subject Classification. Primary 53C44, 53C55; Secondary 55R25.

Key words and phrases. Kähler-Ricci flow, singularity analysis, projective bundles.

The author was supported in part by NSF Grant DMS-\#0604960.

(C) 2013 American Mathematical Society Reverts to public domain 28 years from publication 
in the study of algebraic varieties by Song, Tian, Weinkove et. al in [ST1, T, ST3, SW2, SW3, SSW]. The unified theme of these works is the conjecture that the Kähler-Ricci flow will carry out an analytic analogue of Mori's minimal model program which is about searching for birationally equivalent models that are "minimal" in some algebraic sense. Like Hamilton-Perelman's work, a surgery may need to be performed by continuing the flow if necessary. To this end, the Gromov-Hausdorff convergence provides a bridge to continue the relevant geometric data.

For a better understanding of singularity formation of the Kähler-Ricci flow, one could study some algebraically concrete spaces and explore their flow behavior and possible singularity types and models. In the work by Feldman-Ilmanen-Knopf FIK], Cao Cao2 and Koiso Koi], gradient Kähler-Ricci solitons were constructed on the $\mathcal{O}(-k)$-bundles over $\mathbb{P}^{n}$. Their work employs the $U(n+1) / \mathbb{Z}_{k}$-symmetry introduced by Calabi in $\mathrm{C}$ which reduces the Kähler-Ricci flow equation to a PDE with one spatial variable. Assuming Calabi's symmetry, Song-Weinkove SW1 characterized the limit behavior (in the Gromov-Hausdorff sense) of the Hirzebruch surfaces $\mathbb{P}(\mathcal{O} \oplus \mathcal{O}(-k))$ and their higher dimensional analogues, which are $\mathbb{P}^{1}$-bundles over $\mathbb{P}^{n}$. In their paper, it was proved that the Kähler-Ricci flow exhibits three distinct behaviors: (1) collapsing along the $\mathbb{P}^{1}$-fibers; (2) contracting the exceptional divisor; or (3) shrinking to a point. This trichotomy is determined by the triple $\left(n, k,\left[\omega_{0}\right]\right)$, where $\left[\omega_{0}\right]$ is the initial Kähler class. Later in [SW2, case (2) is much more generalized and the assumption on the symmetry is removed. The Calabi symmetry assumption is removed in case (1) by a recent preprint [SSW] by Song, Székelyhidi and Weinkove.

The purpose of this paper is two-fold. For one thing, we generalize SongWeinkove's work [SW1] on Hirzebruch surfaces to a class of projective bundles over any compact Kähler-Einstein manifold. We will employ an ansatz, known as the momentum construction, which coincides with Calabi's $U(n+1) / \mathbb{Z}_{k}$-symmetry on Hirzebruch surfaces where the base manifold has the Fubini-Study metric. The idea of the momentum construction of projective bundles was introduced and studied in the subject of extremal Kähler metrics by Hwang-Singer in $\mathrm{HS}$ and by ApostolovCalderbank-Gauduchon-(Tønnesen-Friedman) in ACGT. We will show that under this momentum construction, one can give a cohomological criteria under which the Kähler-Ricci flow will collapse the $\mathbb{P}^{1}$-fiber near the singularity similar to the Hirzebruch surface cases in [SW1. Secondly, we study the singularity model of these projective bundles (including Hirzebruch surfaces) via the techniques developed by Hamilton in [H3]. We show that these collapsing projective bundles equipped with momenta will all exhibit $\mathbb{C}^{n} \times \mathbb{P}^{1}$-singularities, and also that the Ricci flow solution has a Type I singularity. Here is the summary of our results:

Main results. Let $M=\mathbb{P}\left(\mathcal{O}_{\Sigma} \oplus L\right)$ be a projective bundle where $\left(\Sigma, \omega_{\Sigma}\right)$ is a compact Kähler-Einstein manifold such that $\operatorname{Ric}\left(\omega_{\Sigma}\right)=\nu \omega_{\Sigma}$ for some $\nu \in \mathbb{R}$, and $L \rightarrow \Sigma$ is a holomorphic line bundle that admits a Hermitian metric $h$ such that the Chern curvature is given by $F_{\nabla}=-\lambda \omega_{\Sigma}, \lambda>0$. Let $\omega_{0}$ be a Kähler metric on $M$ constructed by a $U(1)$-invariant momentum profile with Kähler class $\left[\omega_{0}\right]=$ $\lambda b_{0}\left[\Sigma_{\infty}\right]-\lambda a_{0}\left[\Sigma_{0}\right]$. Suppose the triple $\left(\Sigma, L,\left[\omega_{0}\right]\right)$ satisfies the following conditions:

$$
\begin{aligned}
& \nu \leq \lambda \text { or } \\
& \nu>\lambda \text { and }(\nu-\lambda) b_{0}<(\nu+\lambda) a_{0} .
\end{aligned}
$$


Then along the Kähler-Ricci flow $\partial_{t} \omega_{t}=-\operatorname{Ric}\left(\omega_{t}\right), t \in[0, T)$, we have

- $(M, g(t))$ converges in the Gromov-Hausdorff sense to $\left(\Sigma, \omega_{\Sigma}\right)$ (Theorem 5.4);

- the associated ancient $\kappa$-solution is $\mathbb{C}^{n} \times \mathbb{P}^{1}$ (Theorem 7.3);

- the Ricci flow solution must have a Type I singularity (Theorem [7.4).

This paper is organized as follows. Sections 2 and 3 are the preliminaries which define our projective bundles and construct Kähler metrics using momentum profiles. We will see that the Kähler-Ricci flow is equivalent to a heat-type equation for the evolving momentum profile. Section 4 explains the trichotomy of blow-up exhibited by a different choice of the triples $\left(\Sigma, L,\left[\omega_{0}\right]\right)$ via the calculation of Kähler classes and Chern classes. Section 5 is a variation on the theme of Song-Weinkove's work [SW1] on Hirzebruch surfaces (the collapsing case). We show that similar limiting behavior can be observed in our projective bundles. Sections 6 and 7 are about singularity analysis using rescaled dilations. We show in Section 6 that the ancient $\kappa$-solution obtained from the Cheeger-Gromov limit must split into a product. We will classify their singularity type and the curvature blow-up rate in Section 7 .

We also acknowledge that Kähler-Ricci solitons on this category of bundles (and their variants) were studied and constructed in $\mathrm{DW}, \mathrm{Yg}, \mathrm{Li}]$.

\section{Projective BundLes}

In this section, we will define and elaborate on the projective bundles under consideration in this paper. We first start with a compact Kähler-Einstein manifold $\Sigma^{n}$ with $\operatorname{dim}_{\mathbb{C}}=n$. A Kähler manifold is called Kähler-Einstein if it admits a Kähler form $\omega_{\Sigma}$ whose Ricci form is a real constant multiple of $\omega_{\Sigma}$, i.e. $\operatorname{Ric}\left(\omega_{\Sigma}\right)=\nu \omega_{\Sigma}$, $\nu \in \mathbb{R}$. Clearly, a necessary condition for a compact Kähler manifold to be KählerEinstein is that the first Chern class $c_{1}$ has a definite sign. It is well known by results of Aubin $\left[\mathrm{A}\right.$ and Yau $\left[\mathrm{Y}\right.$ that when $c_{1}<0$ or $=0$, Kähler-Einstein metric always exists. However, if $c_{1}>0$ (i.e. Fano manifolds), Kähler-Einstein metrics do not exist in general. For compact Riemann surfaces, i.e. $\operatorname{dim}_{\mathbb{C}}=1$, the KählerEinstein metric must exist according to the classical uniformization theorem. See also Cheng-Yau's work [CY] on pseudoconvex domains in the complete non-compact case.

In this article, we will not go into the detail of existence issues of Kähler-Einstein metrics, but we will start with a compact Kähler manifold $\Sigma^{n}$ which is equipped with a Kähler-Einstein metric $\omega_{\Sigma}$, such that the Ricci form is given by $\operatorname{Ric}\left(\omega_{\Sigma}\right)=$ $\nu \omega_{\Sigma}$ where $\nu \in \mathbb{R}$. We take this Kähler-Einstein manifold to be our base manifold, and build a projective $\mathbb{P}^{1}$-bundle upon it. Precisely, we construct our projective bundles as follows:

$$
M=\mathbb{P}\left(\mathcal{O}_{\Sigma} \oplus L\right) .
$$

Here $\mathcal{O}_{\Sigma}$ is the trivial line bundle, and $L \rightarrow \Sigma$ is a holomorphic line bundle which is equipped with a Hermitian-Einstein metric $h$ such that $\sqrt{-1} \partial \bar{\partial} \log h=\lambda \omega_{\Sigma}, \lambda \in \mathbb{R}$. Here $\mathbb{P}$ denotes the projectivization of the holomorphic rank-2 bundle $\mathcal{O}_{\Sigma} \oplus L$ over $\Sigma$. The local trivialization $(z, u)$ of this rank-2 bundle has transition functions of the form $\left(z_{\alpha}, u_{\alpha}\right) \approx\left(z_{\beta}, \eta_{\alpha \beta} u_{\alpha}\right)$ for some $\eta_{\alpha \beta} \in \check{H}^{1}\left(\Sigma, \mathcal{O}_{\Sigma}^{*}\right)$. Passing to the projectivization quotient, every element under this trivialization can be expressed as either $\left[1: \frac{u}{z}\right]$ for $z \neq 0$ or $[0: 1]$, and we may regard $[0: 1]$ as the infinity. One can easily check that the projectivization factors through the identification by the transition functions $\mathcal{O}_{\Sigma} \oplus L$. Therefore, one can regard the projectivization of 
$\mathcal{O}_{\Sigma} \oplus L$ as compactifying each fiber by adding an infinity point $(x,[0: 1])$ and hence $M$ can be regarded as a $\mathbb{P}^{1}$-bundle over $\Sigma$. We define $\Sigma_{0}$ to be the zero section $\{x:[1: 0]\}$ and $\Sigma_{\infty}$ to be the infinity section $\{x:[0: 1]\}$. It is easy to see that the zero section $\Sigma_{0}$ and the infinity section $\Sigma_{\infty}$ are global over $\Sigma$.

The class of holomorphic line bundles over $\Sigma$ with tensor product as the operation form a group which is known as the Picard group, denoted by $\operatorname{Pic}(\Sigma)$. For $\Sigma=\mathbb{P}^{n}$, it is well known (see e.g. $\mathrm{GH}$ ) that $\operatorname{Pic}\left(\mathbb{P}^{n}\right)=\mathbb{Z}$ and the line bundles over $\mathbb{P}^{n}$ are given by $\mathcal{O}_{\mathbb{P}^{n}}(k), k \in \mathbb{Z}$. In particular if $(\Sigma, L)=\left(\mathbb{P}^{1}, \mathcal{O}_{\mathbb{P}^{1}}(-k)\right), k>0$, the projective bundles $M=\mathbb{P}\left(\mathcal{O}_{\mathbb{P}^{1}} \oplus \mathcal{O}_{\mathbb{P}^{1}}(-k)\right)$ are called the Hirzebruch surfaces. When $k=1$, the projective bundle is $\mathbb{P}^{2} \#\left(-\mathbb{P}^{2}\right)$, i.e. $\mathbb{P}^{2}$ blown-up at a point. When $\Sigma=\mathbb{C} / \Lambda$, i.e. an elliptic curve or a 2 -torus, the class of line bundles are classified by a classical result by Appell-Humbert (see Mum). In general, for the Riemann surface $\Sigma_{g}$ of genus $g$, the Picard group $\operatorname{Pic}\left(\Sigma_{g}\right)$ is isomorphic to $J\left(\Sigma_{g}\right) \times \mathbb{Z}$ where $J\left(\Sigma_{g}\right)$ is a compact complex manifold $\mathbb{C}^{g} / \Lambda$ of dimension $g$.

The projective bundle $M$ under our consideration is characterized by the pair $(\Sigma, L)$ where $\Sigma$ is a compact Kähler-Einstein manifold and $L$ a holomorphic line bundle over $\Sigma$ which is equipped with a Hermitian metric $h$ such that the Chern curvature is of the form $F_{\nabla}=-\lambda \omega_{\Sigma}$. In particular, the line bundles generated by $\operatorname{det}\left(T^{*} M\right)$ all fall into this category. Moreover, we will only focus on line bundles $L$ with $\lambda>0$, since the projective bundle $\mathbb{P}\left(\mathcal{O}_{\Sigma} \oplus L\right)$ is biholomorphic to its dual cousin $\mathbb{P}\left(\mathcal{O}_{\Sigma} \oplus L^{*}\right)$. Since $c_{1}(L)=-c_{1}\left(L^{*}\right)$, one can replace $L$ by $L^{*}$ in case $c_{1}(L)$ is negative. We do not discuss the case of flat bundles, i.e. $\lambda=0$, in this paper.

\section{U(1)-INVARIANT KÄHLER METRICS}

Let's first recapitulate the construction of the category of projective bundles we are concerned about in the rest of this article. We let $M=\mathbb{P}\left(\mathcal{O}_{\Sigma} \oplus L\right)$, where $\left(\Sigma, \omega_{\Sigma}\right)$ is a Kähler-Einstein manifold such that $\operatorname{Ric}\left(\omega_{\Sigma}\right)=\nu \omega_{\Sigma}, \nu \in \mathbb{R}$. Suppose $L$ is a holomorphic line bundle over $\Sigma$ such that it equips with a Hermitian metric $h$ whose Chern curvature is of the form $F_{\nabla}=-\lambda \omega_{\Sigma}, \lambda>0$. In particular, such a Hermitian metric $h$ must exist if $\omega_{\Sigma}$ is a compact Riemann surface.

We will discuss the construction of $U(1)$-invariant Kähler metrics on these projective bundles in this section. Regard the circle group $U(1)$ as $\left\{e^{i \theta}: \theta \in[0,2 \pi)\right\}$. The $U(1)$-action is defined by

$$
e^{i \theta} \cdot(x,[z: u])=\left(x,\left[z: e^{i \theta} u\right]\right) .
$$

Clearly, the action factors through the transition functions of the bundle, and fixes the zero and infinity sections.

Recall that $\omega_{\Sigma}$ is the Kähler-Einstein form on the manifold $\Sigma$ and we have $\operatorname{Ric}\left(\omega_{\Sigma}\right)=\nu \omega_{\Sigma}$ for some $\nu \in \mathbb{R}$. Using the Hermitian-Einstein metric $h$ described above, one can define a height parameter $\rho$ on $M \backslash\left(\Sigma_{0} \cup \Sigma_{\infty}\right)$ given by

$$
\rho=\log \|\cdot\|_{h}^{2} .
$$

Note that $\rho=-\infty$ corresponds to the zero section and $\rho=\infty$ corresponds to the infinity section.

Our next step is to define Kähler metrics on $M$ which are invariant under the circle action defined above. We start by looking for possible Kähler classes that $M$ can have. We denote $\left[\Sigma_{0}\right]$ and $\left[\Sigma_{\infty}\right]$ as the Poincaré duals (with respect to a fixed background volume form) of $\Sigma_{0}$ and $\Sigma_{\infty}$ in $H_{2}(M, \mathbb{R})$ respectively, i.e. $\int_{\Sigma_{\infty}}\left[\Sigma_{\infty}\right]=-\int_{\Sigma_{0}}\left[\Sigma_{0}\right]=1$. We look for Kähler metrics whose Kähler classes have 
the form $b \lambda\left[\Sigma_{\infty}\right]-a \lambda\left[\Sigma_{0}\right]$ with $b>a>0$. Note also that $c_{1}(L)=[-\sqrt{-1} \partial \bar{\partial} \rho]=$ $-\lambda \pi^{*}\left[\omega_{\Sigma}\right]=\lambda\left[\Sigma_{0}\right]-\lambda\left[\Sigma_{\infty}\right]$.

In order to define a Kähler metric in the Kähler class $b\left[\Sigma_{\infty}\right]-a\left[\Sigma_{0}\right]$, we first define a momentum profile function $f(\rho)$ on $M_{0}=M \backslash\left(\Sigma_{0} \cup \Sigma_{\infty}\right)$. The idea of this momentum construction comes from the works [HS] by Hwang-Singer and ACGT by Apostolov-Calderbank-Gauduchon-(Tønnesen-Friedman) on extremal and constant scalar curvature Kähler metrics. Together with a pair of asymptotic conditions given below, one can extend the metric induced by $f$ to the whole manifold $M$. Here are the details:

Let $f(\rho): \mathbb{R} \rightarrow(a, b)$ be a strictly increasing function. We define a Kähler metric $\omega$ on $M_{0}$ by

$$
\omega=f(\rho) \sqrt{-1} \partial \bar{\partial} \rho+\sqrt{-1} f_{\rho}(\rho) \partial \rho \wedge \partial \bar{\rho} .
$$

Remark 3.1. If we let $u(\rho)$ be the anti-derivative of $f$, i.e. $u_{\rho}=f$, then one can check that $\omega=\sqrt{-1} \partial \bar{\partial} u(\rho)$ on $M_{0}$.

In order for the Kähler metric to be defined on $M$, we require the following asymptotic conditions:

(1) There exists a smooth function $F_{0}:[0, \infty) \rightarrow \mathbb{R}$ with $F_{0}(0)=a$ and $F_{0}^{\prime}(0)>0$, so that $f(\rho)=F_{0}\left(e^{2 \rho}\right)$ as $\rho \rightarrow-\infty$.

(2) There exists a smooth function $F_{\infty}:[0, \infty) \rightarrow \mathbb{R}$ with $F_{\infty}(0)=b$ and $F_{\infty}^{\prime}(0)>0$ so that $f(\rho)=F_{\infty}\left(e^{-2 \rho}\right)$ as $\rho \rightarrow \infty$.

Note that $f$ has to be a strictly increasing function, so we have $a<f(\rho)<b$ for $\rho \in$ $\mathbb{R}$, and

$$
\begin{array}{r}
\lim _{\rho \rightarrow-\infty} f(\rho)=a, \\
\lim _{\rho \rightarrow \infty} f(\rho)=b . \\
\lim _{\rho \rightarrow \pm \infty} f_{\rho}(\rho)=0 .
\end{array}
$$

The Kähler class $[\omega]$ can be easily seen to be $[\omega]=b \lambda\left[\Sigma_{\infty}\right]-a \lambda\left[\Sigma_{0}\right]$, because

$$
\begin{aligned}
\left\langle[\omega], \Sigma_{\infty}\right\rangle & =\int_{\Sigma_{\infty}} b[\sqrt{-1} \partial \bar{\partial} \rho] \\
& =\int_{\Sigma_{\infty}} b \lambda\left[\Sigma_{\infty}\right]=b \lambda \\
\left\langle[\omega], \Sigma_{0}\right\rangle & =\int_{\Sigma_{0}} a[\sqrt{-1} \partial \bar{\partial} \rho] \\
& =\int_{\Sigma_{0}}-a \lambda\left[\Sigma_{0}\right]=a \lambda .
\end{aligned}
$$

Under this construction, the Kähler form depends only on the height parameter $\rho$. We can see immediately that these Kähler metrics are invariant under the $U(1)$ action defined earlier, since the action preserves $\rho:\left\|e^{i \theta} u\right\|_{h}=\|u\|_{h}$ for any section $u \in \Gamma(\Sigma, L)$.

Note that for $(\Sigma, L)=\left(\mathbb{P}^{n}, \mathcal{O}_{\mathbb{P}^{n}}(-k)\right)$, i.e. Hirzebruch-type manifolds, the above momentum construction with $\omega_{\Sigma}=\omega_{\mathrm{FS}}$, i.e. the Fubini-Study metric, is the $U(n+1) / \mathbb{Z}_{k}$-symmetry initiated by Calabi in [C].

Next we derive the local expression of the Kähler metric $\omega$ constructed by the above momentum profile as well as its Ricci curvature. Let $\left(z_{1}, \ldots, z_{n}, \xi\right)$ be local 
holomorphic coordinates of $M$ where $\mathbf{z}=\left(z_{1}, \ldots, z_{n}\right)$ are the base coordinates and $\xi$ is the fiber coordinate. Recall that the height parameter is defined to be

$$
\rho=\log \|\cdot\|_{h}^{2} .
$$

Let $\phi(\mathbf{z})$ be a positive function such that $\|\xi\|^{2}=|\xi|^{2} \phi(\mathbf{z})$ for any $(\mathbf{z}, \xi)$ in the local coordinate chart. Then we have

$$
\rho=\log |\xi|^{2}+\log \phi(\mathbf{z}) .
$$

Using this, one can easily check $\rho_{\xi \bar{\xi}}=\rho_{i \bar{\xi}}=\rho_{\xi \bar{i}}=0$ for any $i=1,2, \ldots, n$. Moreover, $\sqrt{-1} \partial \bar{\partial} \rho=\lambda \pi^{*} \omega_{\Sigma}$, so we can let $\lambda \pi^{*} \omega_{\Sigma}=\rho_{i \bar{j}} d z^{i} \wedge d \bar{z}^{j}$. Hence, the Kähler metric in $(\mathbf{z}, \xi)$ coordinates is given by

$$
\begin{aligned}
\omega= & \sqrt{-1} \sum_{i, j=1}^{n}\left(f \rho_{i \bar{j}}+f_{\rho} \rho_{i} \rho_{\bar{j}}\right) d z^{i} \wedge d z^{\bar{j}}+\sqrt{-1} f_{\rho} \sum_{i=1}^{n} \rho_{i} \rho_{\bar{\xi}} d z^{i} \wedge d \bar{\xi} \\
& +\sqrt{-1} f_{\rho} \sum_{i=1}^{n} \rho_{\xi} \rho_{\bar{i}} d \xi \wedge d z^{\bar{i}}+\sqrt{-1} f_{\rho}\left|\rho_{\xi}\right|^{2} d \xi \wedge d \bar{\xi} .
\end{aligned}
$$

Let $g$ be the metric associated to the Kähler form $\omega$, and $g_{\Sigma}$ be that of $\omega_{\Sigma}$. The determinant of the metric $g$ and its logarithm are given by

$$
\begin{aligned}
\operatorname{det}(g) & =\lambda^{n} f^{n} f_{\rho} \operatorname{det}\left(g_{\Sigma}\right)|\xi|^{-2}, \\
\log \operatorname{det}(g) & =n \log \lambda+n \log f+\log f_{\rho}+\log \operatorname{det}\left(g_{\Sigma}\right)-\log |\xi|^{2} .
\end{aligned}
$$

Using this, one can then compute the Ricci form $-\sqrt{-1} \partial \bar{\partial} \log \operatorname{det}(g)$ :

$$
\begin{aligned}
\operatorname{Ric}(\omega)= & -\sqrt{-1} \partial \bar{\partial} \log \operatorname{det}(g) \\
= & \left\{\left(\nu \lambda^{-1}-\partial_{\rho}\left(n \log f+\log f_{\rho}\right)\right) \rho_{i \bar{j}}-\partial_{\rho \rho}\left(n \log f+\log f_{\rho}\right) \rho_{i} \rho_{\bar{j}}\right\} d z^{i} \wedge d z^{\bar{j}} \\
& -\partial_{\rho \rho}\left(n \log f+\log f_{\rho}\right) \rho_{i} \rho_{\bar{\xi}} d z^{i} \wedge d \bar{\xi}-\partial_{\rho \rho}\left(n \log f+\log f_{\rho}\right) \rho_{\xi} \rho_{\bar{i}} d \xi \wedge d z^{\bar{i}} \\
& -\partial_{\rho \rho}\left(n \log f+\log f_{\rho}\right)\left|\rho_{\xi}\right|^{2} d \xi \wedge d \bar{\xi} .
\end{aligned}
$$

In the computation of the Ricci form, we used the fact that $\omega_{\Sigma}$ is Kähler-Einstein so that $-\sqrt{-1} \partial \bar{\partial} \log \operatorname{det}\left(g_{\Sigma}\right)=\nu \omega_{\Sigma}$.

Observing that the $\omega$ and Ric have similar linear-algebraic expressions when $\omega$ is constructed by a momentum profile $f$, one can easily see that the Kähler-Ricci flow on $M$ is equivalent to a parabolic equation that evolves the momentum profile. In other words, the Kähler-Ricci flow preserves the momentum construction. Precisely, we have

Proposition 3.2. Suppose $\omega_{0}$ is the initial Kähler form on $M$ with momentum profile $f_{0}(\rho)$. Then the solution $\omega_{t}, t \in[0, T)$, to the Kähler-Ricci flow $\partial_{t} \omega_{t}=$ $-\operatorname{Ric}\left(\omega_{t}\right)$ also admits a momentum profile $f(\rho, t)$ at each time $t \in[0, T)$ where $f(\rho, t)$ evolves by

$$
\frac{\partial f}{\partial t}=\frac{\partial}{\partial \rho}\left(n \log f(\rho, t)+\log f_{\rho}(\rho, t)\right)-\frac{\nu}{\lambda}, \quad f(\rho, 0)=f_{0}(\rho),
$$

or equivalently,

$$
\frac{\partial f}{\partial t}=\frac{f_{\rho \rho}}{f_{\rho}}+n \frac{f_{\rho}}{f}-\frac{\nu}{\lambda}, \quad f(\rho, 0)=f_{0}(\rho)
$$




\section{KÄHLER CLASSES UNDER KÄHLER-RICCI FLOW}

From now on, we will consider the Kähler-Ricci flow $\partial_{t} \omega_{t}=-\operatorname{Ric}\left(\omega_{t}\right)$ on $M$ which satisfies the aforesaid $U(1)$-symmetry and admits evolving momenta $f(\rho, t)$. We say $T$ is the blow-up time of the Ricci flow if $[0, T)$ is the maximal time interval for the Ricci flow to exist. For Ricci flow on compact Kähler manifolds, the blow-up time is completely determined by the initial Kähler class and the first Chern class. Namely, we have the following theorem proved by Tian-Zhang:

Theorem 4.1 (Tian-Zhang, [TZ]). Let $(X, \omega(t))$ be an (unnormalized) KählerRicci flow $\partial_{t} \omega_{t}=-\operatorname{Ric}\left(\omega_{t}\right)$ on a compact Kähler manifold $X^{n}$. Then the blow-up time $T$ is given by

$$
T=\sup \left\{t:\left[\omega_{0}\right]+t c_{1}\left(K_{X}\right)>0\right\}
$$

where $K_{X}:=\operatorname{det}\left(T^{*} X\right)$ is the canonical line bundle of $X^{n}$.

Note that the Kähler class $\left[\omega_{t}\right]$ at any time $t$ is given by $\left[\omega_{t}\right]=\left[\omega_{0}\right]+t c_{1}\left(K_{X}\right)$. In order to work out the evolving Kähler classes and the blow-up time, one needs to understand the first Chern class of $K_{X}$, which can be computed by the adjunction formula.

Given any smooth divisor $D$ of compact Kähler manifold $X$, the adjunction formula relates $K_{X}$ and $K_{D}$ by

$$
K_{D}=\left.\left(K_{X} \otimes \mathcal{N}_{M / D}\right)\right|_{D},
$$

where $\mathcal{N}_{M / D}$ is the normal bundle of $D$ in $M$.

Using (4.1), one can easily work out $c_{1}\left(K_{M}\right)$ by taking $D=\Sigma_{0}, \Sigma_{\infty}$ in turn. For example, taking $D=\Sigma_{\infty}$, we have

$$
\begin{aligned}
K_{\Sigma_{\infty}} & =\left.\left(K_{M} \otimes L^{*}\right)\right|_{\Sigma_{\infty}}, \\
\left\langle c_{1}\left(K_{\Sigma_{\infty}}\right),\left[\Sigma_{\infty}\right]\right\rangle & =\left\langle c_{1}\left(K_{M}\right)-c_{1}(L),\left[\Sigma_{\infty}\right]\right\rangle .
\end{aligned}
$$

Since $\Sigma$ is Kähler-Einstein such that $\operatorname{Ric}\left(\omega_{\Sigma}\right)=\nu \omega_{\Sigma}$, we then have

$$
\left\langle c_{1}\left(K_{\Sigma_{\infty}}\right),\left[\Sigma_{\infty}\right]\right\rangle=-\nu .
$$

Since $c_{1}(L)=\lambda\left[\Sigma_{0}\right]-\lambda\left[\Sigma_{\infty}\right]$, we have $\left\langle c_{1}(L),\left[\Sigma_{\infty}\right]\right\rangle=-\lambda$, and hence

$$
\left\langle c_{1}\left(K_{M}\right),\left[\Sigma_{\infty}\right]\right\rangle=-\nu-\lambda \text {. }
$$

Similarly, one can also show by taking $D=\Sigma_{0}$ in (4.1) (now $\mathcal{N}_{M \backslash D}=L$ ) to show

$$
\left\langle c_{1}\left(K_{M}\right),\left[\Sigma_{0}\right]\right\rangle=-\nu+\lambda .
$$

Therefore, the first Chern class of the canonical line bundle $K_{M}$ is given by:

$$
c_{1}\left(K_{M}\right)=(-\nu-\lambda)\left[\Sigma_{\infty}\right]-(-\nu+\lambda)\left[\Sigma_{0}\right] .
$$

Hence, under the Kähler-Ricci flow $\partial_{t} \omega_{t}=-\operatorname{Ric}\left(\omega_{t}\right)$ with initial class $\left[\omega_{0}\right]=$ $b_{0} \lambda\left[\Sigma_{\infty}\right]-a_{0} \lambda\left[\Sigma_{0}\right]$, the Kähler class evolves by

$$
\left[\omega_{t}\right]=\left(b_{0} \lambda-(\nu+\lambda) t\right)\left[\Sigma_{\infty}\right]-\left(a_{0} \lambda-(\nu-\lambda) t\right)\left[\Sigma_{0}\right] .
$$

We denote $\left[\omega_{t}\right]=\lambda b_{t}\left[\Sigma_{\infty}\right]-\lambda a_{t}\left[\Sigma_{0}\right]$ where $a_{t}, b_{t}$ are defined by

$$
\begin{aligned}
a_{t} & :=a_{0}-\frac{(\nu-\lambda)}{\lambda} t, \\
b_{t} & :=b_{0}-\frac{(\nu+\lambda)}{\lambda} t .
\end{aligned}
$$


Note also that $\left[\pi^{*} \omega_{\Sigma}\right]=\left[\Sigma_{\infty}\right]-\left[\Sigma_{0}\right]$, therefore the Kähler class can also be expressed as

$$
\left[\omega_{t}\right]=\lambda a_{t}\left[\pi^{*} \omega_{\Sigma}\right]+\lambda\left(b_{t}-a_{t}\right)\left[\Sigma_{\infty}\right] .
$$

Hence, by Theorem 4.1, the maximal time is characterized by $\lambda$ and $\nu$ in the following way:

- Case 1: $\nu \leq \lambda$

In this case, $\left[\omega_{t}\right]$ ceases to be Kähler when $b_{t}=a_{t}$, namely, at $T:=\frac{b_{0}-a_{0}}{2}$. The limiting Kähler class is given by

$$
\left[\omega_{T}\right]=\lambda a_{T}\left[\pi^{*} \omega_{\Sigma}\right]
$$

This holds true for any given $b_{0}>a_{0}>0$.

- Case 2: $\nu>\lambda$

We further divide it into three sub-cases

(i) $(\nu-\lambda) b_{0}<(\nu+\lambda) a_{0}$ :

$\left[\omega_{t}\right]$ ceases to be Kähler when $b_{t}=a_{t}$. Likewise, the limiting Kähler class is given by

$$
\left[\omega_{T}\right]=\lambda a_{T}\left[\pi^{*} \omega_{\Sigma}\right]
$$

(ii) $(\nu-\lambda) b_{0}=(\nu+\lambda) a_{0}$ :

$\left[\omega_{t}\right]$ is then proportional to $c_{1}\left(K_{M}^{-1}\right)$, i.e. the canonical class. The flow stops at $T=\frac{a_{0} \lambda}{\nu-\lambda}$ and the limiting class $\left[\omega_{T}\right]=0$. It is well known (see e.g. [ST2] that in such a case $(M, g(t))$ extincts and converges to a point in the Gromov-Hausdorff sense as $t \rightarrow T$.

(iii) $(\nu-\lambda) b_{0}>(\nu+\lambda) a_{0}$ :

$\left[\omega_{t}\right]$ ceases to be Kähler when at $T=a_{0}$, and the limit class is given by $\left[\omega_{T}\right]=\lambda b_{T}\left[\Sigma_{\infty}\right]$.

This trichotomy resembles that in Song-Weinkove's work [SW1 on Hirzebruch surfaces and Hirzebruch-type manifolds, i.e. $(\Sigma, L)=\left(\mathbb{P}^{n}, \mathcal{O}_{\mathbb{P}^{n}} \oplus \mathcal{O}_{\mathbb{P}^{n}}(-k)\right)$. In their work, from which our study was motivated, similar trichotomy of the blow-up time of the Kähler-Ricci flow with initial Kähler class $\left[\omega_{0}\right]$ was also exhibited, as it is characterized by the triple $\left(n, k,\left[\omega_{0}\right]\right)$. It was shown in [SW1] assuming Calabi's $U(n+1) / \mathbb{Z}_{k}$-symmetry and in [SSW] assuming $\Sigma$ is projective that in the case of having limiting Kähler class $a_{T}\left[\pi^{*} \omega_{\Sigma}\right]$, the Kähler-Ricci flow collapses the $\mathbb{P}^{1}$-fiber of the projective bundle, which hereof converges to some Kähler metric of $\Sigma$ as metric spaces in the Gromov-Hausdorff sense.

Case 2(iii) is reminiscent of Song-Weinkove's recent works [SW2 and SW3 of contracting exceptional divisors, in which the $\mathcal{O}(-k)$-blow-up of the arbitrary compact Kähler manifold $X$ are considered. In their works, a cohomological condition is given on the initial Kähler class and the first Chern class, under which the blownup manifold will converge in the Gromov-Hausdorff sense back to $X$ with orbifold singularity of type $\mathcal{O}(-k)$. There is no symmetry assumption in these works.

In our paper, we will only focus on Case 1 and Case 2(i) which exhibit a collapsing of the $\mathbb{P}^{1}$-fiber assuming the Kähler metric admits the aforesaid momentum construction. 


\section{Estimates OF THE KÄHLER-RiCCI FLOW}

From now on we assume that the triple $\left(\Sigma, L,\left[\omega_{0}\right]\right)$ satisfies Case 1 or Case 2(i) stated in the previous section, i.e. either

$$
\begin{aligned}
& \nu \leq \lambda \text { or } \\
& \nu>\lambda \text { and }(\nu-\lambda) b_{0}<(\nu+\lambda) a_{0} .
\end{aligned}
$$

Recall that $\nu$ is the Ricci curvature of the Kähler-Einstein manifold $\Sigma$ and $\lambda$ is the Chern curvature of the Hermitian-Einstein line bundle $L$, i.e.

$$
\begin{aligned}
\operatorname{Ric}\left(\omega_{\Sigma}\right) & =\nu \omega_{\Sigma}, \\
\sqrt{-1} \partial \bar{\partial} \rho & =-\lambda \pi^{*} \omega_{\Sigma} .
\end{aligned}
$$

Recall that the first Chern class of $K_{M}$ and the evolving Kähler class are given by:

$$
\begin{aligned}
c_{1}\left(K_{M}\right) & =(-\nu-\lambda)\left[\Sigma_{\infty}\right]-(-\nu+\lambda)\left[\Sigma_{0}\right] \\
& =(-\nu+\lambda)\left[\pi^{*} \omega_{\Sigma}\right]-2 \lambda\left[\Sigma_{\infty}\right], \\
{\left[\omega_{t}\right] } & =\lambda b_{t}\left[\Sigma_{\infty}\right]-\lambda a_{t}\left[\Sigma_{0}\right] \\
& =\lambda a_{t}\left[\pi^{*} \omega_{\Sigma}\right]+2 \lambda(T-t)\left[\Sigma_{\infty}\right]
\end{aligned}
$$

where $a_{t}$ and $b_{t}$ are defined in (4.3) and (4.4).

Since pluripotential theory plays a very important role in Kähler-Ricci flow and in Kähler geometry in general, we would like to understand the Kähler-Ricci flow $\partial_{t} \omega_{t}=-\operatorname{Ric}\left(\omega_{t}\right)$ from the viewpoint of potential functions. To do so, we need a reference family of Kähler metrics $\left\{\hat{\omega}_{t}\right\}_{t \in[0, T)}$ whose Kähler class at each time $t$ coincides with that of $\omega_{t}$, the Kähler-Ricci flow solution. We choose $\hat{\omega}_{t}$ to be the $U(1)$-invariant Kähler metric induced by the following momentum profile:

$$
\hat{f}(\rho, t):=a_{t}+\frac{\left(b_{t}-a_{t}\right) e^{2 \rho}}{1+e^{2 \rho}}=a_{t}+\frac{2 \lambda(T-t) e^{2 \rho}}{1+e^{2 \rho}} .
$$

This momentum profile gives the following Kähler metric:

$$
\hat{\omega}_{t}=a_{t} \sqrt{-1} \partial \bar{\partial} \rho+2 \sqrt{-1} \lambda(T-t)\left(\frac{e^{2 \rho}}{1+e^{2 \rho}} \partial \bar{\partial} \rho+\frac{2 e^{2 \rho}}{\left(1+e^{2 \rho}\right)^{2}} \partial \rho \wedge \bar{\partial} \rho\right) .
$$

Clearly, $\hat{f}$ satisfies the asymptotic conditions for extending $\hat{\omega}_{t}$ to the whole $M$. Also, we have $\left[\hat{\omega}_{t}\right]=\left[\omega_{t}\right]$ because $\hat{f} \rightarrow a_{t}$ as $\rho \rightarrow-\infty$ and $\hat{f} \rightarrow b_{t}$ as $\rho \rightarrow \infty$.

For simplicity, we denote $\Theta:=\frac{e^{2 \rho}}{1+e^{2 \rho}} \sqrt{-1} \partial \bar{\partial} \rho+\frac{2 e^{2 \rho}}{\left(1+e^{2 \rho}\right)^{2}} \sqrt{-1} \partial \rho \wedge \bar{\partial} \rho$, so that

$$
\hat{\omega}_{t}=a_{t} \pi^{*} \omega_{\Sigma}+2 \lambda(T-t) \Theta .
$$

Note that $[\Theta]=\left[\Sigma_{\infty}\right]$ and so $\frac{\partial \hat{\omega}_{t}}{\partial t}=(-\nu+\lambda) \pi^{*} \omega_{\Sigma}-2 \lambda \Theta \in c_{1}\left(K_{M}\right)$. Take $\Omega$ to be a fixed volume form of $M$ such that $\frac{\partial \hat{\omega}_{t}}{\partial t}=\sqrt{-1} \partial \bar{\partial} \log \Omega$. Then the Kähler-Ricci flow $\partial_{t} \omega_{t}=-\operatorname{Ric}\left(\omega_{t}\right)$ is equivalent to the following complex Monge-Ampère equation:

$$
\frac{\partial \phi}{\partial t}=\log \frac{\operatorname{det}\left(\hat{\omega}_{t}+\sqrt{-1} \partial \bar{\partial} \phi\right)}{(T-t) \Omega},\left.\quad \phi\right|_{t=0}=\phi_{0}
$$

in a sense that $\omega_{t}=\hat{\omega}_{t}+\sqrt{-1} \partial \bar{\partial} \phi, t \in[0, T)$, is a solution to the Kähler-Ricci flow $\partial_{t} \omega_{t}=-\operatorname{Ric}\left(\omega_{t}\right)$ with initial data $\omega_{0}=\hat{\omega}_{0}+\sqrt{-1} \partial \bar{\partial} \phi_{0}$ if and only if $\phi: M \times[0, T)$ is a solution to (5.1).

Working similarly as in [ST1, SW1, SW2, TZ], etc., one can derive the following estimates using maximum principles. 
Lemma 5.1. There exists a constant $C=C\left(n, \omega_{0}, \nu, \lambda\right)>1$ such that the following holds:

(1) $|\phi(t)| \leq C$,

(2) $\omega_{t}^{n+1} \leq C \Omega$, and

(3) $\operatorname{Tr}_{\omega_{t}} \pi^{*} \omega_{\Sigma} \leq C$.

Proof. The proof goes similarly as in [SW1]. First note that since

$$
\hat{\omega}_{t}^{n+1} \geq 2 \lambda a_{t}(n+1)(T-t)\left(\pi^{*} \omega_{\Sigma}\right)^{n} \wedge \Theta,
$$

one can then find a constant $C>0$ independent of $t$ such that

$$
C^{-1}(T-t) \Omega \leq \hat{\omega}_{t}^{n+1} \leq C(T-t) \Omega .
$$

Consider the function $\tilde{\phi}=\phi+(1+\log C) t$; at the point $p_{t} \in M$ where $\tilde{\phi}$ achieves its minimum at time $t$, we have $\partial \bar{\partial} \tilde{\phi}=\partial \bar{\partial} \phi \geq 0$. Therefore,

$$
\begin{aligned}
\frac{d}{d t} \tilde{\phi}_{\min }(t) & =\left.\log \frac{\operatorname{det}\left(\hat{\omega}_{t}+\sqrt{-1} \partial \bar{\partial} \phi\right)}{(T-t) \Omega}\right|_{p_{t}}+\log C \\
& \geq\left.\log \frac{\operatorname{det} \hat{\omega}_{t}}{C^{-1}(T-t) \Omega}\right|_{p_{t}} \geq 0 .
\end{aligned}
$$

Here we used (5.2). It proves $\phi$ is uniformly bounded from below as the flow encounters finite-time singularity. The uniform upper bound for $\phi$ follows similarly.

For (2), we consider $Q:=\frac{\partial \phi}{\partial t}-|\lambda-\nu| a^{-1} \phi+\log (T-t)$ where $a:=\inf _{[0, T)} a_{t}>0$. By direct computation, we have

$$
\begin{aligned}
\frac{\partial Q}{\partial t}= & \operatorname{Tr}_{\omega_{t}}\left((\lambda-\nu) \pi^{*} \omega_{\Sigma}-2 \lambda \Theta\right)+\Delta\left(\frac{\partial \phi}{\partial t}\right) \\
& -|\lambda-\nu| a^{-1}\left(Q+|\lambda-\nu| a^{-1} \phi-\log (T-t)\right)-\frac{1}{T-t} \\
= & \Delta Q+|\lambda-\nu| a^{-1} \Delta \phi+(\lambda-\nu) \operatorname{Tr}_{\omega_{t}} \pi^{*} \omega_{\Sigma}-2 \lambda \operatorname{Tr}_{\omega_{t}} \Theta \\
& -|\lambda-\nu| a^{-1}\left(Q+|\lambda-\nu| a^{-1} \phi-\log (T-t)\right)-\frac{1}{T-t} .
\end{aligned}
$$

Since $\omega_{t}=a_{t} \pi^{*} \omega_{\Sigma}+2 \lambda(T-t) \Theta+\sqrt{-1} \partial \bar{\partial} \phi(t)$, taking trace with respect to $\omega_{t}$ yields

$$
n+1=a_{t} \operatorname{Tr}_{\omega_{t}} \pi^{*} \omega_{\Sigma}+2 \lambda(T-t) \operatorname{Tr}_{\omega_{t}} \Theta+\Delta \phi \geq a_{t} \operatorname{Tr}_{\omega_{t}} \pi^{*} \omega_{\Sigma}+\Delta \phi .
$$

Hence we have

$$
|\lambda-\nu| a^{-1} \Delta \phi \leq|\lambda-\nu| a^{-1}(n+1)-|\lambda-\nu| \operatorname{Tr}_{\omega_{t}} \pi^{*} \omega_{\Sigma}
$$

Note that $a_{t} \geq a$ for any $t \in[0, T)$. Combining (5.3) and (5.4), we have

$$
\square Q \leq(n+1)|\lambda-\nu| a^{-1}-|\lambda-\nu| a^{-1}\left(Q+|\lambda-\nu| a^{-1} \phi\right)+|\lambda-\nu| a^{-1} \log T .
$$

As $\phi$ is uniformly bounded from (1), (5.5) implies a uniformly upper bound for $Q$. Since $Q=\log \frac{\operatorname{det} \omega_{t}}{\Omega}-|\lambda-\nu| a^{-1} \phi$, again together with the uniform bound for $\phi$, we proved (2).

Finally, for $(3)$, we let $\left(z_{1}, \ldots, z_{n}, \xi\right)$ be local holomorphic coordinates such that $\mathbf{z}=\left(z_{1}, \ldots, z_{n}\right)$ is the base coordinate and $\xi$ is the fiber coordinate. Then the bundle map is given by $\pi:(\mathbf{z}, \xi) \mapsto \mathbf{z}$. Write $\lambda \pi^{*} \omega_{\Sigma}=\rho_{i \bar{j}} d z^{i} \wedge d \bar{z}^{j}$. Assuming the 
Kähler metric $\omega_{t}$ admits momentum profiles $f(\rho, t)$, we have $g^{i \bar{j}}=\frac{1}{f} \rho^{i \bar{j}}$ and one can prove

$$
\operatorname{Tr}_{\omega_{t}} \pi^{*} \omega_{\Sigma}=\frac{1}{\lambda} g^{i \bar{j}} \rho_{i \bar{j}}=\frac{n}{\lambda f}
$$

which is clearly bounded from above uniformly independent of $t$.

Next, we will derive estimates on the Kähler-Ricci flow by assuming the metric is $U(1)$-invariant and admits a momentum profile $f(\rho, t)$. First note that because $f_{\rho}(\rho, t)>0$ for any $t$ and also $\lim _{\rho \rightarrow-\infty} f(\rho, t)=a_{t}, \lim _{\rho \rightarrow \infty} f(\rho, t)=b_{t}$, we have

$$
a_{t}<f(\rho, t)<b_{t}, \quad \text { for any }(\rho, t) \in \mathbb{R} \times[0, T) .
$$

Note that $a_{t}$ and $b_{t}$ are both bounded away from zero as $t \rightarrow T ;(2)$ in Lemma 5.1 implies $f_{\rho}$ is also uniformly bounded. Using these, one is able to derive the following estimates.

Lemma 5.2. There exists a constant $C=C\left(n, \omega_{0}, \nu, \lambda\right)>0$ such that

(1) $C^{-1} \leq f \leq C$,

(2) $\left|\frac{f_{\rho \rho}}{f_{\rho}}\right| \leq C$,

(3) $f_{\rho} \leq C(T-t)$

for any $(\rho, t) \in \mathbb{R} \times[0, T)$.

Proof. As discussed above, (1) clearly holds because $a_{t}$ is bounded away from zero and $b_{t}$ is uniformly bounded above on $[0, T)$.

For (2), first note that by the asymptotic conditions of the momentum profile $f(\rho, t), \lim _{\rho \rightarrow \pm \infty}\left|\frac{f_{\rho \rho}}{f_{\rho}}\right|=2$ for any $t \in[0, T)$, so $\sup _{\mathbb{R} \times[0, T-\epsilon)}\left|\frac{f_{\rho \rho}}{f_{\rho}}\right|$ exists for every $\epsilon>0$. We will derive the uniform lower bound for $\frac{f_{\rho \rho}}{f_{\rho}}$ on $[0, T)$ since the upper bound is similar. Given any $\epsilon>0$, let $\left(\rho_{\epsilon}, t_{\epsilon}\right) \in \mathbb{R} \times[0, T-\epsilon)$ be the point such that

$$
\left.\frac{f_{\rho \rho}}{f_{\rho}}\right|_{\left(\rho_{\epsilon}, t_{\epsilon}\right)}=\sup _{\mathbb{R} \times[0, T-\epsilon)} \frac{f_{\rho \rho}}{f_{\rho}} .
$$

Then at $\left(\rho_{\epsilon}, t_{\epsilon}\right)$, one has $\frac{\partial}{\partial t}\left(\frac{f_{\rho \rho}}{f_{\rho}}\right) \geq 0, \frac{\partial}{\partial \rho}\left(\frac{f_{\rho \rho}}{f_{\rho}}\right)=0$, and $\frac{\partial^{2}}{\partial \rho^{2}}\left(\frac{f_{\rho \rho}}{f_{\rho}}\right) \leq 0$.

Recall that $f$ satisfies the heat-type equation (3.2), i.e. $\frac{\partial f}{\partial t}=\frac{f_{\rho \rho}}{f_{\rho}}+n \frac{f_{\rho}}{f}-\frac{\nu}{\lambda}$. By direct computation, one has

$$
\begin{aligned}
\frac{\partial}{\partial t}\left(\frac{f_{\rho \rho}}{f_{\rho}}\right) & =\frac{2 n f_{\rho}^{2}}{f^{3}}-\frac{2 n f_{\rho \rho}}{f^{2}}-\frac{n f_{\rho \rho}^{2}}{f f_{\rho}^{2}}+\frac{3 f_{\rho \rho}^{3}}{f_{\rho}^{4}}+\frac{n f_{\rho \rho \rho}}{f f_{\rho}}-\frac{4 f_{\rho \rho} f_{\rho \rho \rho}}{f_{\rho}^{3}}+\frac{f_{\rho \rho \rho \rho}}{f_{\rho}^{2}}, \\
\frac{\partial}{\partial \rho}\left(\frac{f_{\rho \rho}}{f_{\rho}}\right) & =\frac{f_{\rho} f_{\rho \rho \rho}-f_{\rho \rho}^{2}}{f_{\rho}^{2}} \\
\frac{\partial^{2}}{\partial \rho^{2}}\left(\frac{f_{\rho \rho}}{f_{\rho}}\right) & =\frac{2 f_{\rho \rho}^{3}}{f_{\rho}^{3}}-\frac{3 f_{\rho \rho} f_{\rho \rho \rho}}{f_{\rho}^{2}}+\frac{f_{\rho \rho \rho \rho}}{f_{\rho}} .
\end{aligned}
$$

Evaluating at $\left(\rho_{\epsilon}, t_{\epsilon}\right)$, the fact that $\frac{\partial}{\partial \rho}\left(\frac{f_{\rho \rho}}{f_{\rho}}\right)=0$ implies $f_{\rho \rho \rho}=\frac{f_{\rho \rho}^{2}}{f_{\rho}}$ at $\left(\rho_{\epsilon}, t_{\epsilon}\right)$. By substituting $f_{\rho \rho \rho}=\frac{f_{\rho \rho}^{2}}{f_{\rho}}$ into the expressions of $\frac{\partial}{\partial t}\left(\frac{f_{\rho \rho}}{f_{\rho}}\right)$ and $\frac{\partial^{2}}{\partial \rho^{2}}\left(\frac{f_{\rho \rho}}{f_{\rho}}\right)$, one can check that after cancellation of terms, we have

$$
0 \leq\left(\frac{\partial}{\partial t}-\frac{1}{f_{\rho}} \frac{\partial^{2}}{\partial \rho^{2}}\right) \frac{f_{\rho \rho}}{f_{\rho}}=\frac{2 n f_{\rho}^{2}}{f^{3}}-\frac{2 n f_{\rho \rho}}{f^{2}} \quad \text { at }\left(\rho_{\epsilon}, t_{\epsilon}\right) .
$$


It shows $\sup _{\mathbb{R} \times[0, T-\epsilon)} \frac{f_{\rho \rho}}{f_{\rho}}=\left.\frac{f_{\rho \rho}}{f_{\rho}}\right|_{\left(\rho_{\epsilon}, t_{\epsilon}\right)} \leq\left.\frac{f_{\rho}}{f}\right|_{\left(\rho_{\epsilon}, t_{\epsilon}\right)}$. Since $f_{\rho}$ is uniformly bounded from above and $f>C^{-1}$, there exists $C>0$ independent of $\epsilon$ such that

$$
\sup _{\mathbb{R} \times[0, T-\epsilon)} \frac{f_{\rho \rho}}{f_{\rho}} \leq C .
$$

A similar approach proves $\inf _{\mathbb{R} \times[0, T)} \frac{f_{\rho \rho}}{f_{\rho}} \geq-\tilde{C}$ for some uniform constant $\tilde{C}>0$. It completes the proof of (2).

Part (3) follows from part (2). Precisely, (2) implies $\left|\left(\log f_{\rho}\right)_{\rho}\right| \leq C$. If we let $\rho_{t} \in \mathbb{R}$ such that $f_{\rho}\left(\rho_{t}\right)=\sup _{\rho \in \mathbb{R}} f_{\rho}$, then by the mean-value theorem,

$$
\left|\log f_{\rho}(\rho, t)-\log f_{\rho}\left(\rho_{t}, t\right)\right| \leq C\left|\rho-\rho_{t}\right| .
$$

Thus for $\rho \in\left[\rho_{t}-C^{-1}, \rho_{t}+C^{-1}\right]$, we have

$$
\log \left(\frac{f_{\rho}(\rho, t)}{f_{\rho}\left(\rho_{t}, t\right)}\right) \geq-1,
$$

or equivalently, $f_{\rho}(\rho, t) \geq e^{-1} f_{\rho}\left(\rho_{t}, t\right)$. We then have

$$
\int_{\mathbb{R}} f_{\rho} d \rho \geq \int_{\rho_{t}-C^{-1}}^{\rho_{t}+C^{-1}} f_{\rho} d \rho \geq 2 C^{-1} e^{-1} f_{\rho}\left(\rho_{t}, t\right) .
$$

On the other hand, we have

$$
\int_{\mathbb{R}} f_{\rho} d \rho=f(\infty)-f(-\infty)=b_{t}-a_{t}=2 \lambda(T-t) .
$$

Hence $\sup _{\rho \in \mathbb{R}} f_{\rho} \leq C(T-t)$ for some uniform constant $C$.

Lemma 5.2 implies that the $\mathbb{P}^{1}$-fiber of our manifold $M$ is collapsing along the flow. Precisely we have the following:

Proposition 5.3. Assume $\left(\Sigma, L,\left[\omega_{0}\right]\right)$ satisfies the condition stated in Case 1 and Case 2(i) following Theorem 4.1. Let $V_{x} \in T_{x} M$ be a tangent vector of $M$ at $x \in M \backslash\left(\Sigma_{0} \cup \Sigma_{\infty}\right)$ which lies $T_{x} \mathbb{P}_{x}^{1}$. Here we denote $\mathbb{P}_{x}^{1}$ as the $\mathbb{P}^{1}$-fiber passing through $x$. Then we have $\left\|V_{x}\right\|_{g(t)} \rightarrow 0$ as $t \rightarrow T$.

Proof. It suffices to express $\left\|V_{x}\right\|_{g(t)}$ in terms of $f$ and $f_{\rho}$, since the metric $g(t)$ is given by

$$
g(t)=f \lambda \pi^{*} g_{\Sigma}+f_{\rho} \partial \rho \otimes \bar{\partial} \rho .
$$

Since $V_{x}$ is parallel to the fiber, we have $\pi_{*} V_{x}=0$ and so $\pi^{*} g_{\Sigma}\left(V_{x}, \bar{V}_{x}\right)=0$. Hence $\left\|V_{x}\right\|_{g(t)}^{2}=f_{\rho} \frac{\partial V_{x}}{\partial \rho} \frac{\partial \bar{V}_{x}}{\partial \rho} \rightarrow 0$ as $t \rightarrow T$. Here we have used part (3) of Lemma 5.2.

Furthermore, Lemmas 5.1 and 5.2 provide enough estimates in order to show $\left(M, \omega_{t}\right)$ converges to $\left(\Sigma, a_{T} \omega_{\Sigma}\right)$ as metric spaces in the Gromov-Hausdorff sense.

Theorem 5.4. Suppose $\left(\Sigma, L,\left[\omega_{0}\right]\right)$ satisfies the condition stated in Case 1 and Case 2(i) following Theorem 4.1, then $(M, g(t))$ converges to the Kähler-Einstein manifold $\left(\Sigma, a_{T} \omega_{\Sigma}\right)$ in the Gromov-Hausdorff sense as $t \rightarrow T$.

Proof. The proof proceeds in almost the same manner as in Song-Weinkove's paper SW1 on Hirzebruch surfaces with Calabi ansatz. We will sketch the main idea 
here. For details, please refer to Song-Weinkove's paper. The main ingredients of the argument are as follows:

(1) the metric $g(t)$ is degenerating along the fiber direction on compact subsets of $M \backslash\left(\Sigma_{0} \cup \Sigma_{\infty}\right)$,

(2) $g(t)$ is bounded above uniformly $g(0)$, and

(3) for any $0<\alpha<1, g(t)$ converges to $a_{T} \pi^{*} \omega_{\Sigma}$ in the $C^{\alpha}$-sense on compact subsets of $M \backslash\left(\Sigma_{0} \cup \Sigma_{\infty}\right)$.

Proposition 5.3 implies (1). (2) can be proved by a uniform estimate on $f_{\rho}$ which can be easily obtained by the bound on the volume form $\omega_{t}^{n+1}$ in Lemma 5.1, For $(3)$, note that $\omega_{t}=f(\rho, t) \sqrt{-1} \partial \bar{\partial} \rho+\sqrt{-1} f_{\rho}(\rho, t) \partial \rho \wedge \bar{\partial} \rho$. One can compute that $\left\|\nabla_{g_{0}} g(t)\right\|_{g_{0}}^{2}$ is a polynomial expression of $f(\rho, t), f_{\rho}(\rho, t)$ and $f_{\rho \rho}(\rho, t)$ where the coefficients are time-independent. Lemma 5.2 then shows for any compact subset $K \in M \backslash\left(\Sigma_{0} \cup \Sigma_{\infty}\right)$, so we have $\sup _{K \times[0, T)}\left\|\nabla_{g_{0}} g(t)\right\|_{g_{0}}^{2} \leq C_{K}$ for some time-independent constant $C_{K}>0$. It proves $(3)$.

To show the Gromov-Hausdorff convergence, first fix a leave of $\Sigma$ in $M \backslash\left(\Sigma_{0} \cup\right.$ $\left.\Sigma_{\infty}\right)$. We denote it by $\sigma(\Sigma)$. Using $(2)$, one can choose a sufficiently small tubular neighborhood of $\Sigma_{0}$ and $\Sigma_{\infty}$ such that their complement contains $\sigma(\Sigma)$. Then given any two points $x_{1}, x_{2} \in M$, we project them down to the base $\Sigma$ via the bundle map $\pi$. Consider the length of the geodesic $\gamma$ joining $\pi\left(x_{1}\right)$ and $\pi\left(x_{2}\right)$; by lifting the geodesic up by $\sigma$, we know that the lifted $\gamma$ has length arbitrarily close to the $a_{T} \omega_{\Sigma^{-}}$ length by (3). Finally, using (1), one can show $x_{i}$ is arbitrarily close to $\sigma \circ \pi\left(x_{i}\right)$ as $t \rightarrow T$. Using triangle inequality, one can then prove that the $g(t)$-distance between $x_{1}$ and $x_{2}$ is arbitrarily close to the $a_{T} \omega_{\Sigma}$-distance as $t \rightarrow T$.

\section{SPlitTing LEMMA}

In the singularity analysis of closed (real) 3-manifolds as in [H3] and [P1], one often considers a rescaled dilation, which is a rescaled sequence of metrics $g_{i}(t)=K_{i} g\left(t_{i}+K_{i}^{-1} t\right)$ where $K_{i}$ are chosen such that $K_{i}=\left\|\operatorname{Rm}\left(x_{i}\right)\right\|_{g\left(t_{i}\right)} \rightarrow \infty$ and $\left\|\mathrm{Rm}_{g_{i}(t)}\right\|_{g_{i}(t)} \leq C$ for some uniform constant $C>0$ independent of $i$. By Hamilton's compactness $\mathrm{H} 3$ and Perelman's local non-collapsing theorem $\mathrm{P} 1$, one can extract a subsequence, still call it $g_{i}(t)$, such that $\left(M, g_{i}(t), x_{i}\right) \rightarrow\left(M_{\infty}, g_{\infty}(t), x_{\infty}\right)$ on compact subsets in the Cheeger-Gromov sense. The convergence is in $C^{\infty}$ topology because once the curvature tensor is uniformly bounded, Shi's derivative estimate in [Shi] asserts all the higher order derivatives of Rm are uniformly bounded. The limit obtained is often called a singularity model. According to the curvature blow-up rate (Type I or II), a singularity model may be an ancient or eternal solution, and is $\kappa$-non-collapsed by Perelman's result. These singularity models encode crucial geometric data near the singularity region of the flow.

We will show that under our momentum construction and our assumption on the triple $\left(\Sigma, L,\left[\omega_{0}\right]\right)$, the singularity model $M_{\infty}$ obtained by the aforesaid rescaled dilations splits isometrically into a product $N \times L$, where $\operatorname{dim}_{\mathbb{C}} N=n$ and $\operatorname{dim}_{\mathbb{C}} L=$ 1 .

Let $\left(z_{1}, \ldots, z_{n}, \xi\right)$ be local holomorphic coordinates where $z=\left(z_{1}, \ldots, z_{n}\right)$ are the base coordinates and $\xi$ is the fiber coordinate. Then $\lambda \pi^{*} \omega_{\Sigma}=\sqrt{-1} \rho_{i \bar{j}}(z) d z^{i} \wedge d z^{\bar{j}}$, the Kähler metric defined by momentum profile $f(\rho, t)$, its inverse and the Ricci 
tensor are locally written as

$$
\begin{gathered}
g_{A B}= \begin{cases}f \rho_{i \bar{j}}+f_{\rho} \rho_{i} \rho_{\bar{j}} & \text { if }(A, B)=(i, \bar{j}), \\
f_{\rho} \rho_{i} \rho_{\bar{\xi}} & \text { if }(A, B)=(i, \bar{\xi}), \\
f_{\rho}\left|\rho_{\xi}\right|^{2} & \text { if }(A, B)=(\xi, \bar{\xi}),\end{cases} \\
g^{A B}= \begin{cases}\frac{1}{f} \rho^{i \bar{j}} & \text { if }(A, B)=(i, \bar{j}), \\
-\frac{1}{f \rho_{\bar{\xi}}} \sum_{k=1}^{n} \rho^{i \bar{k}} \rho_{\bar{k}} & \text { if }(A, B)=(i, \bar{\xi}), \\
\frac{1}{\left|\rho_{\xi}\right|^{2}}\left(\frac{1}{f_{\rho}}+\frac{\sum_{k, l=1}^{n} \rho^{k \bar{l}} \rho_{k} \rho_{\bar{l}}}{f}\right) & \text { if }(A, B)=(\xi, \bar{\xi}),\end{cases} \\
\operatorname{Ric}_{A B}= \begin{cases}\left(\nu \lambda^{-1}-F_{\rho}\right) \rho_{i \bar{j}}-F_{\rho \rho} \rho_{i} \rho_{\bar{j}} & \text { if }(A, B)=(i, \bar{j}), \\
-F_{\rho \rho} \rho_{i} \rho_{\bar{\xi}} & \text { if }(A, B)=(i, \bar{\xi}), \\
-F_{\rho \rho}\left|\rho_{\xi}\right|^{2} & \text { if }(A, B)=(\xi, \bar{\xi}),\end{cases}
\end{gathered}
$$

where $F=n \log f+\log f_{\rho}$.

From the local expressions of $g$ and $g^{-1}$, one can easily derive local expressions of the Christoffel symbols which we will need for deriving our splitting result.

Lemma 6.1. The Christoffel symbols of the Kähler metric $g$ on $M$ constructed by momentum profile $f$ are given by

$$
\begin{aligned}
\Gamma_{\xi \xi}^{i} & =0, \\
\Gamma_{\xi \xi}^{\xi} & =\frac{f_{\rho \rho}}{f_{\rho}} \rho_{\xi}+\frac{\rho_{\xi \xi}}{\rho_{\xi}}=\left(\frac{f_{\rho \rho}}{f_{\rho}}-1\right) \rho_{\xi}, \\
\Gamma_{i \xi}^{\xi} & =\left(\frac{f_{\rho \rho}}{f_{\rho}}-\frac{f_{\rho}}{f}\right) \rho_{i}, \\
\Gamma_{i \xi}^{j} & =\frac{f_{\rho}}{f} \delta_{i}^{j} \rho_{\xi}, \\
\Gamma_{i j}^{\xi} & =\left(\frac{f_{\rho \rho}}{f_{\rho}}-\frac{2 f_{\rho}}{f}\right) \frac{\rho_{i} \rho_{j}}{\rho_{\xi}}-\frac{1}{\rho_{\xi}}\left(\rho^{l \bar{k}} \rho_{l} \rho_{j \bar{k} i}+\rho_{i j}\right), \\
\Gamma_{i j}^{k} & =\frac{f_{\rho}}{f}\left(\rho_{i} \delta_{j}^{k}+\rho_{j} \delta_{i}^{k}\right)+\rho^{k \bar{l}} \rho_{j \overline{l i}} .
\end{aligned}
$$

Remark 6.2. Recall that for Kähler manifolds, the only (possibly) non-zero Christoffel symbols are those with indexes of either all (1,0)-type or all $(0,1)$-type. For succinctness, please excuse us for omitting those which are vanishing or conjugate to one of the above.

Remark 6.3. We will see that the vanishing of $\Gamma_{\xi \xi}^{i}$ is crucial when dealing with the curvature tensor in the blow-up analysis in the next section. Moreover, we only need the first four Christoffel symbols in order to obtain the splitting lemma.

Proof. Using the formula $\Gamma_{\alpha \beta}^{\gamma}=g^{\gamma \bar{\delta}} \partial_{\alpha} g_{\beta \bar{\delta}}$ for Kähler manifolds, one can compute the Christoffel symbols directly:

$$
\begin{aligned}
\Gamma_{\xi \xi}^{i} & =g^{i \bar{j}} \frac{\partial}{\partial \xi} g_{\xi \bar{j}}+g^{i \bar{\xi}} \frac{\partial}{\partial \xi} g_{\xi \bar{\xi}} \\
& =\frac{1}{f} \rho^{i \bar{j}} \frac{\partial}{\partial \xi}\left(f_{\rho} \rho_{\xi} \rho_{\bar{j}}\right)-\frac{1}{f \rho_{\bar{\xi}}} \rho^{i \bar{k}} \rho_{\bar{k}} \frac{\partial}{\partial \xi}\left(f_{\rho} \rho_{\xi} \rho_{\bar{\xi}}\right)
\end{aligned}
$$




$$
\begin{aligned}
& =\frac{1}{f} \rho^{i \bar{j}}\left(f_{\rho \rho} \rho_{\xi} \rho_{\xi} \rho_{\bar{j}}+f_{\rho} \rho_{\xi \xi} \rho_{\bar{j}}\right)-\frac{1}{f \rho_{\bar{\xi}}} \rho^{i \bar{k}} \rho_{\bar{k}}\left(f_{\rho \rho} \rho_{\xi} \rho_{\xi} \rho_{\bar{\xi}}+f_{\rho} \rho_{\xi \xi} \rho_{\bar{\xi}}\right) \\
& =0 \text {, } \\
& \Gamma_{\xi \xi}^{\xi}=\sum_{i=1}^{n} g^{\xi \bar{i}} \frac{\partial}{\partial \xi} g_{\xi \bar{i}}+g^{\xi \bar{\xi}} \frac{\partial}{\partial \xi} g_{\xi \bar{\xi}} \\
& =-\frac{1}{f \rho_{\xi}} \sum_{k=1}^{n} \rho^{k \bar{i}} \rho_{k} \frac{\partial}{\partial \xi}\left(f_{\rho} \rho_{\xi} \rho_{\bar{i}}\right)+\frac{1}{\left|\rho_{\xi}\right|^{2}}\left(\frac{1}{f_{\rho}}+\frac{\sum_{k, l=1}^{n} \rho^{k \bar{l}} \rho_{k} \rho_{\bar{l}}}{f}\right) \frac{\partial}{\partial \xi}\left(f_{\rho} \rho_{\xi} \rho_{\bar{\xi}}\right) \\
& =-\frac{1}{f \rho_{\xi}} \sum_{k=1}^{n} \rho^{k \bar{i}} \rho_{k} \frac{\partial}{\partial \xi}\left(f_{\rho \rho} \rho_{\xi}^{2} \rho_{\bar{i}}+f_{\rho} \rho_{\xi \xi} \rho_{\bar{i}}\right) \\
& +\frac{1}{\left|\rho_{\xi}\right|^{2}}\left(\frac{1}{f_{\rho}}+\frac{\sum_{k, l=1}^{n} \rho^{k \bar{l}} \rho_{k} \rho_{\bar{l}}}{f}\right)\left(f_{\rho \rho} \rho_{\xi}^{2} \rho_{\bar{\xi}}+f_{\rho} \rho_{\xi \xi} \rho_{\bar{\xi}}\right) \\
& =\frac{f_{\rho \rho}}{f_{\rho}} \rho_{\xi}+\frac{\rho_{\xi \xi}}{\rho_{\xi}}=\left(\frac{f_{\rho \rho}}{f_{\rho}}-1\right) \rho_{\xi}, \\
& \Gamma_{i \xi}^{\xi}=\sum_{j=1}^{n} g^{\xi \bar{j}} \frac{\partial}{\partial z_{i}} g_{\xi \bar{j}}+g^{\xi \bar{\xi}} \frac{\partial}{\partial z_{i}} g_{\xi \bar{\xi}} \\
& =-\frac{1}{f \rho_{\xi}} \rho^{k \bar{j}} \rho_{k} \frac{\partial}{\partial z_{i}}\left(f_{\rho} \rho_{\xi} \rho_{\bar{j}}\right)+\frac{1}{\left|\rho_{\xi}\right|^{2}}\left(\frac{1}{f_{\rho}}+\frac{\sum_{k, l=1}^{n} \rho^{k \bar{l}} \rho_{k} \rho_{\bar{l}}}{f}\right) \frac{\partial}{\partial z_{i}}\left(f_{\rho} \rho_{\xi} \rho_{\bar{\xi}}\right) \\
& =-\frac{1}{f \rho_{\xi}} \rho^{k \bar{j}} \rho_{k}\left(f_{\rho \rho} \rho_{i} \rho_{\xi} \rho_{\bar{j}}+f_{\rho} \rho_{\xi} \rho_{i \bar{j}}\right) \\
& +\frac{1}{\left|\rho_{\xi}\right|^{2}}\left(\frac{1}{f_{\rho}}+\frac{\sum_{k, l=1}^{n} \rho^{k \bar{l}} \rho_{k} \rho_{\bar{l}}}{f}\right)\left(f_{\rho \rho} \rho_{i} \rho_{\xi} \rho_{\bar{\xi}}\right) \\
& =\left(\frac{f_{\rho \rho}}{f_{\rho}}-\frac{f_{\rho}}{f}\right) \rho_{i}, \\
& \Gamma_{i \xi}^{j}=\sum_{k=1}^{n} g^{j \bar{k}} \frac{\partial}{\partial z_{i}} g_{\xi \bar{k}}+g^{j \xi} \frac{\partial}{\partial z_{i}} g_{\xi \bar{\xi}} \\
& =\frac{1}{f} \rho^{j \bar{k}} \frac{\partial}{\partial z_{i}}\left(f_{\rho} \rho_{\xi} \rho_{\bar{k}}\right)-\frac{1}{f \rho_{\bar{\xi}}} \rho^{j \bar{k}} \rho_{\bar{k}} \frac{\partial}{\partial z_{i}}\left(f_{\rho} \rho_{\xi} \rho_{\bar{\xi}}\right) \\
& =\frac{1}{f} \rho^{j \bar{k}}\left(f_{\rho \rho} \rho_{i} \rho_{\xi} \rho_{\bar{k}}+f_{\rho} \rho_{\xi} \rho_{i \bar{k}}\right)-\frac{1}{f \rho_{\bar{\xi}}} \rho^{j \bar{k}} \rho_{\bar{k}}\left(f_{\rho \rho} \rho_{i} \rho_{\xi} \rho_{\bar{\xi}}\right) \\
& =\frac{f_{\rho}}{f} \delta_{i}^{j} \rho_{\xi}, \\
& \Gamma_{i j}^{\xi}=\sum_{k=1}^{n} g^{\xi \bar{k}} \frac{\partial}{\partial z_{i}} g_{j \bar{k}}+g^{\xi \bar{\xi}} \frac{\partial}{\partial z_{i}} g_{j \bar{\xi}} \\
& =-\frac{1}{f \rho_{\xi}} \rho^{\bar{k} l} \rho_{l} \frac{\partial}{\partial z_{i}}\left(f \rho_{j \bar{k}}+f_{\rho} \rho_{j} \rho_{\bar{k}}\right)+\frac{1}{\left|\rho_{\xi}\right|^{2}}\left(\frac{1}{f_{\rho}}+\frac{\rho^{k \bar{l}} \rho_{k} \rho_{\bar{l}}}{f}\right) \frac{\partial}{\partial z_{i}}\left(f_{\rho} \rho_{j} \rho_{\bar{\xi}}\right) \\
& =-\frac{f_{\rho}}{f \rho_{\xi}} \rho^{l \bar{k}} \rho_{l}\left(\rho_{i} \rho_{j \bar{k}}+\rho_{j} \rho_{i \bar{k}}\right)-\frac{1}{\rho_{\xi}} \rho^{l \bar{k}} \rho_{j \bar{k} i} \rho_{l}+\frac{f_{\rho \rho}}{f_{\rho} \rho_{\xi}} \rho_{i} \rho_{\bar{j}}+\frac{1}{\rho_{\xi}} \rho_{i \bar{j}}
\end{aligned}
$$




$$
\begin{aligned}
= & \left(\frac{f_{\rho \rho}}{f_{\rho}}-\frac{2 f_{\rho}}{f}\right) \frac{\rho_{i} \rho_{j}}{\rho_{\xi}}-\frac{1}{\rho_{\xi}}\left(\rho^{l \bar{k}} \rho_{l} \rho_{j \bar{k} i}+\rho_{i j}\right) \\
\Gamma_{i j}^{k}= & \sum_{k=1}^{n} g^{k \bar{l}} \frac{\partial}{\partial z_{i}} g_{j \bar{l}}+g^{k \bar{\xi}} \frac{\partial}{\partial z_{i}} g_{j \bar{\xi}} \\
= & \frac{1}{f} \rho^{k \bar{l}}\left(f_{\rho} \rho_{i} \rho_{j \bar{l}}+f \rho_{j \bar{l}}+f_{\rho \rho} \rho_{i} \rho_{j} \rho_{\bar{l}}+f_{\rho} \rho_{i j} \rho_{\bar{l}}+f_{\rho} \rho_{j} \rho_{i \bar{l}}\right) \\
& -\frac{1}{f \rho_{\bar{\xi}}} \rho^{k \bar{l}} \rho_{\bar{l}}\left(f_{\rho \rho} \rho_{i} \rho_{j} \rho_{\bar{\xi}}+f_{\rho} \rho_{i j} \rho_{\bar{\xi}}\right) \\
= & \frac{f_{\rho}}{f}\left(\rho_{i} \delta_{j}^{k}+\rho_{j} \delta_{i}^{k}\right)+\rho^{k \bar{l}} \rho_{j \overline{l i}} .
\end{aligned}
$$

Let's state and prove our splitting lemma.

Lemma 6.4. Let $M=\mathbb{P}\left(\mathcal{O}_{\Sigma} \oplus L\right)$ be the projective bundle such that the triple $\left(\Sigma, L,\left[\omega_{0}\right]\right)$ satisfies the assumptions stated in the subsection on main results. Let $\left(M, \omega_{t}\right), t \in[0, T)$ be the Kähler-Ricci flow $\partial_{t} \omega_{t}=-\operatorname{Ric}\left(\omega_{t}\right)$ with initial Kähler class $\left[\omega_{0}\right]$. Let $\left(x_{i}, t_{i}\right) \in M \times[0, T)$ be a sequence such that $t_{i} \rightarrow T$ and $K_{i}:=$ $\left\|\operatorname{Rm}\left(x_{i}\right)\right\|_{g\left(t_{i}\right)} \rightarrow \infty$ as $i \rightarrow \infty$. Define $g_{i}(t)$ to be a rescaled dilated sequence by $K_{i}$ and $t_{i}$, i.e.

$$
g_{i}(t):=K_{i} g\left(t_{i}+K_{i}^{-1} t\right), \quad t \in\left[-\beta_{i}, \alpha_{i}\right]
$$

where $\beta_{i} \rightarrow \infty, \alpha_{i} \geq 0$ and $\alpha_{i} \rightarrow A \in[0, \infty]$. Suppose the curvature tensor of $g_{i}(t), t \in\left[-\beta_{i}, \alpha_{i}\right]$, is uniformly bounded independent of $i$, i.e. there exists $C>0$ independent of $i$ such that

$$
\sup _{M \times\left[-\beta_{i}, \alpha_{i}\right]}\|\mathrm{Rm}\|_{g_{i}(t)} \leq C .
$$

Then, after passing to a subsequence, $\left(M^{n+1}, g_{i}(t), x_{i}\right)$ converges smoothly in the pointed Cheeger-Gromov sense to a complete ancient Kähler-Ricci flow $\left(M_{\infty}, g_{\infty}(t)\right.$, $\left.x_{\infty}\right)$ whose universal cover is of the form

$$
\left(N_{1}^{n} \times N_{2}^{1}, h_{1}(t) \oplus h_{2}(t)\right), \quad t \in(-\infty, A],
$$

where $\left(N_{i}, h_{i}(t)\right), i=1,2$, are Kähler-Ricci flow solutions.

Proof. By the uniform boundedness condition of $\|\mathrm{Rm}\|_{g_{i}(t)}$ over $M \times\left[-\beta_{i}, \alpha_{i}\right]$, the subsequential Cheeger-Gromov convergence can be done by Hamilton's compactness theorem and Perelman's local non-collapsing theorem. See [CCG, H3, P1], etc. Furthermore, we may assume the complex structure of $J$ of $M$ converges after passing to a subsequence to a complex structure $J_{\infty}$ of $M_{\infty}$. That makes $\left(M_{\infty}, J_{\infty}\right)$ Kähler because $\nabla^{g_{\infty}} J_{\infty}=\lim _{i \rightarrow \infty} \nabla^{g_{i}} J=0$.

We will use the well-known de Rham holonomy splitting theorem, which asserts that if the tangent bundle $T M_{\infty}$ admits an irreducible decomposition $\bigoplus_{i=1}^{k} E_{i}$ under the holonomy group action, i.e. parallel translation, then the universal cover of $M_{\infty}$ splits isometrically as $\left(M_{\infty}, g\right)=\prod_{i=1}^{k} N_{i}^{\operatorname{dim} E_{i}}$ with $T N_{i}^{\operatorname{dim} E_{i}}=E_{i}$. Note that in the Kähler case where the holonomy group is a subgroup of the unitary group, each $N_{i}$ is also Kähler.

Suppose $\left(M_{\infty}, g_{\infty}(t), x_{\infty}\right)$ is the pointed Cheeger-Gromov limit obtained above. We would like to show that it (precisely, the universal cover) splits isometrically into a product. According to the nature of the collapsing of the $\mathbb{P}^{1}$-fiber, it is natural 
to guess that one factor of the split product should correspond to the base and the other should correspond to the fiber. Based on these, we define the following unit vector fields:

$$
\begin{aligned}
Z_{g_{i}(t)}^{j} & :=\frac{1}{\left\|\frac{\partial}{\partial z_{j}}\right\|_{g_{i}(t)}} \frac{\partial}{\partial z_{j}}=\frac{1}{\sqrt{K_{i}\left(f \rho_{j \bar{j}}+f_{\rho}\left|\rho_{j}\right|^{2}\right)}} \frac{\partial}{\partial z_{j}}, \\
\Xi_{g_{i}(t)} & :=\frac{1}{\sqrt{K_{i} f_{\rho} \rho_{\xi}}} \frac{\partial}{\partial \xi} .
\end{aligned}
$$

Then we have $\left\|Z_{g_{i}(t)}^{j}\right\|_{g_{i}(t)}=\left\|\Xi_{g_{i}(t)}\right\|_{g_{i}(t)}=1$. After passing to a subsequence, they converge to vector fields $Z_{g_{\infty}(t)}^{j}$ and $\Xi_{g_{\infty}(t)}$ in the limit $M_{\infty}$.

We will show that the real distribution $E_{\infty}=\operatorname{span}_{\mathbb{R}}\left\{\Re\left(\Xi_{g_{\infty}(t)}\right), \Im\left(\Xi_{g_{\infty}(t)}\right)\right\}$ is invariant under parallel translation. Here $\Re$ and $\Im$ denote the real and imaginary parts respectively. For simplicity, we will denote $Z_{g_{i}(t)}^{j}$ as $Z_{i}^{j}$ and $\Xi_{g_{i}(t)}$ as $\Xi_{i}$ for any $i \in \mathbb{N} \cup\{\infty\}$.

It is worthwhile to note that $E_{\infty}^{\perp}=\operatorname{span}_{\mathbb{R}}\left\{\Re\left(Z_{\infty}^{j}\right), \Im\left(Z_{\infty}^{j}\right)\right\}_{j=1}^{n}$ since

$$
\left|\left\langle\Xi_{i}, \bar{Z}_{i}^{j}\right\rangle_{g_{i}(t)}\right|=\left|\frac{1}{\sqrt{K_{i} f_{\rho}} \rho_{\xi}} \cdot \frac{1}{\sqrt{K_{i} g_{j \bar{j}}}} \cdot K_{i} f_{\rho} \rho_{\xi} \rho_{\bar{j}}\right| \leq \sqrt{\frac{f_{\rho}}{f}} \cdot \frac{\left|\rho_{j}\right|}{\sqrt{\rho_{j \bar{j}}}},
$$

which tends to 0 as $i \rightarrow \infty$ using Lemma [5.2. Note that we have used $g_{j \bar{j}}=$ $f \rho_{j \bar{j}}+f_{\rho}\left|\rho_{j}\right|^{2} \geq f \rho_{j \bar{j}}$. Since $\rho=\log |\xi|^{2}+\log \phi(\mathbf{z})$ by (3.1), the term $\frac{\rho_{j}}{\sqrt{\rho_{j \bar{j}}}}$ is independent of $\xi, i$ and $t$, and hence is uniformly bounded near $\rho= \pm \infty$. Therefore $\Xi_{\infty}$ is orthogonal to each of $Z_{\infty}^{j}$, i.e. $E_{\infty}^{\perp}=\operatorname{span}_{\mathbb{R}}\left\{\Re\left(Z_{\infty}^{j}\right), \Im\left(Z_{\infty}^{j}\right)\right\}_{j=1}^{n}$.

In order to show $E_{\infty}$ is invariant under parallel translation, we need to show that by parallel translating $\Xi_{\infty}$ along any vector field $X$ on $M_{\infty}$, it stays inside $E_{\infty}$. We will prove this by showing $\nabla_{X}^{\infty} \Xi_{\infty}$ lies inside $E_{\infty}$, or equivalently, orthogonal to $E_{\infty}^{\perp}$. We will make use of the Christoffel symbols calculated in Lemma 6.1,

$$
\begin{aligned}
\nabla_{\Xi_{i}} \Xi_{i} & =\frac{1}{\sqrt{K_{i} f_{\rho}} \rho_{\xi}} \nabla_{\xi}\left(\frac{1}{\sqrt{K_{i} f_{\rho}} \rho_{\xi}} \frac{\partial}{\partial \xi}\right) \\
& =\frac{1}{\sqrt{K_{i} f_{\rho}} \rho_{\xi}}\left(\frac{\partial}{\partial \xi}\left(\frac{1}{\sqrt{K_{i} f_{\rho}} \rho_{\xi}}\right) \frac{\partial}{\partial \xi}+\frac{1}{\sqrt{K_{i} f_{\rho}} \rho_{\xi}}\left(\Gamma_{\xi \xi}^{\xi} \frac{\partial}{\partial \xi}+\Gamma_{\xi \xi}^{j} \frac{\partial}{\partial z_{j}}\right)\right) \\
& =\frac{1}{\sqrt{K_{i} f_{\rho}} \rho_{\xi}}\left(\frac{1}{\sqrt{K_{i}}}\left(\frac{1}{\sqrt{f_{\rho}}}-\xi \frac{f_{\rho \rho}}{2 f_{\rho}^{3 / 2}} \rho_{\xi}\right) \frac{\partial}{\partial \xi}+\frac{1}{\sqrt{K_{i} f_{\rho}} \rho_{\xi}}\left(\frac{f_{\rho \rho}}{f_{\rho}}-1\right) \rho_{\xi} \frac{\partial}{\partial \xi}\right) \\
& =\frac{1}{K_{i} \sqrt{f_{\rho}} \rho_{\xi}}\left(\frac{1}{\sqrt{f_{\rho}}} \frac{\partial}{\partial \xi}-\frac{f_{\rho \rho}}{2 \sqrt{f_{\rho}} f_{\rho}} \frac{\partial}{\partial \xi}+\frac{1}{\sqrt{f_{\rho}}} \frac{f_{\rho \rho}}{f_{\rho}} \frac{\partial}{\partial \xi}-\frac{1}{\sqrt{f_{\rho}}} \frac{\partial}{\partial \xi}\right) \\
& =\frac{\xi}{2 K_{i} f_{\rho}}\left(\frac{f_{\rho \rho}}{f_{\rho}}\right) \frac{\partial}{\partial \xi} .
\end{aligned}
$$

Taking the inner product with the vectors along the base direction, we have

$$
\begin{aligned}
\left\langle\nabla_{\Xi_{i}} \Xi_{i}, \bar{Z}_{i}^{j}\right\rangle_{g_{i}} & =\frac{\xi}{2 K_{i} f_{\rho}} \cdot \frac{f_{\rho \rho}}{f_{\rho}} \cdot \frac{1}{\sqrt{K_{i}\left(f \rho_{j \bar{j}}+f_{\rho}\left|\rho_{j}\right|^{2}\right)}} \cdot K_{i} f_{\rho} \rho_{\xi} \rho_{\bar{j}}, \\
\left|\left\langle\nabla_{\Xi_{i}} \Xi_{i}, \bar{Z}_{i}^{j}\right\rangle_{g_{i}}\right| & \leq \frac{1}{2 \sqrt{K_{i}}} \cdot \frac{f_{\rho \rho}}{f_{\rho}} \cdot \frac{\left|\rho_{\bar{j}}\right|}{\sqrt{f \rho_{j \bar{j}}}} .
\end{aligned}
$$


Letting $i \rightarrow \infty$, we get $\left\langle\nabla_{\Xi_{\infty}} \Xi_{\infty}, \bar{Z}_{\infty}^{j}\right\rangle_{g_{\infty}}=0$ for any $j=1, \ldots, n$; here $\nabla$ is the Levi-Civita connection with respect to $g_{\infty}$. We have used the estimates proved in Lemma 5.2, which says $f_{\rho \rho} / f_{\rho}=O(1)$ and $f=O(1)$, as well as the fact that $K_{i} \rightarrow \infty$. This proves $\nabla_{\Xi_{\infty}} \Xi_{\infty} \in E_{\infty}$.

Similarly, by parallel translating $\Xi_{\infty}$ along $\bar{\Xi}_{\infty}$, we calculate

$$
\begin{aligned}
\nabla_{\Xi_{i}} \Xi_{i} & =\frac{1}{\sqrt{K_{i} f_{\rho}} \rho_{\bar{\xi}}} \nabla_{\bar{\xi}}\left(\frac{1}{\sqrt{K_{i} f_{\rho}}} \xi \frac{\partial}{\partial \xi}\right) \\
& =-\frac{1}{2 K_{i} f_{\rho}}\left(\frac{f_{\rho \rho}}{f_{\rho}}\right) \xi \frac{\partial}{\partial \xi} \\
& =-\nabla_{\Xi_{i}} \Xi_{i} .
\end{aligned}
$$

Hence, we also have $\left|\left\langle\nabla_{\Xi_{i}} \Xi_{i}, \bar{Z}_{i}^{j}\right\rangle_{g_{i}(t)}\right| \rightarrow 0$ for any $j=1, \ldots, n$ as $i \rightarrow \infty$, and that proves $\left\langle\nabla_{\bar{\Xi}_{\infty}} \Xi_{\infty} \bar{Z}_{\infty}^{j}\right\rangle_{g_{\infty}}=0$ and so $\nabla_{\bar{\Xi}_{\infty}} \Xi_{\infty} \in E_{\infty}$.

The other calculations are similar:

$$
\begin{aligned}
\nabla_{Z_{i}^{j}} \Xi_{i}= & \frac{1}{\sqrt{K_{i} g_{j \bar{j}}}} \nabla_{j}\left(\frac{1}{\sqrt{K_{i} f_{\rho}} \rho_{\xi}} \frac{\partial}{\partial \xi}\right) \\
= & \frac{1}{\sqrt{K_{i} g_{j \bar{j}}}}\left(\frac{\partial}{\partial z_{j}}\left(\frac{1}{\sqrt{K_{i} f_{\rho}} \rho_{\xi}}\right) \frac{\partial}{\partial \xi}+\frac{1}{\sqrt{K_{i} f_{\rho}} \rho_{\xi}}\left(\Gamma_{j \xi}^{k} \frac{\partial}{\partial z_{k}}+\Gamma_{j \xi}^{\xi} \frac{\partial}{\partial \xi}\right)\right) \\
= & \frac{1}{K_{i} \sqrt{g_{j \bar{j}}}}\left(-\frac{\rho_{j}}{2 \sqrt{f_{\rho}} \rho_{\xi}}\left(\frac{f_{\rho \rho}}{f_{\rho}}\right) \frac{\partial}{\partial \xi}\right. \\
& \left.+\frac{1}{\sqrt{f_{\rho}} \rho_{\xi}}\left(\frac{f_{\rho}}{f} \delta_{j}^{k} \rho_{\xi} \frac{\partial}{\partial z_{k}}+\left(\frac{f_{\rho \rho}}{f_{\rho}}-\frac{f_{\rho}}{f}\right) \rho_{j} \frac{\partial}{\partial \xi}\right)\right) \\
= & \frac{1}{K_{i} \rho_{\xi} \sqrt{f_{\rho} g_{j \bar{j}}}}\left(\left(\frac{f_{\rho \rho}}{2 f_{\rho}}-\frac{f_{\rho}}{f}\right) \rho_{j} \frac{\partial}{\partial \xi}+\frac{f_{\rho}}{f} \rho_{\xi} \frac{\partial}{\partial z_{j}}\right), \\
\left\langle\nabla_{Z_{i}^{j}} \Xi_{i}, \bar{Z}_{i}^{k}\right\rangle_{g_{i}(t)}= & \frac{1}{K_{i} \rho_{\xi} \sqrt{f_{\rho} g_{j \bar{j}}} \sqrt{K_{i} g_{k \bar{k}}}} \\
& \times\left\{\left(\frac{f_{\rho \rho}}{2 f_{\rho}}-\frac{f_{\rho}}{f}\right) \rho_{j} K_{i} f_{\rho} \rho_{\xi} \rho_{\bar{k}}+\frac{f_{\rho}}{f} \rho_{\xi} K_{i}\left(f \rho_{j \bar{k}}+f_{\rho} \rho_{j} \rho_{\bar{k}}\right)\right\} \\
= & \sqrt{\frac{f_{\rho}}{K_{i} g_{j \bar{j}} g_{k \bar{k}}}}\left(\frac{f_{\rho \rho}}{2 f_{\rho}} \rho_{j} \rho_{\bar{k}}+\rho_{j \bar{k}}\right) .
\end{aligned}
$$

Hence

$$
\left|\left\langle\nabla_{Z_{i}^{j}} \Xi_{i}, \bar{Z}_{i}^{k}\right\rangle_{g_{i}(t)}\right| \leq \sqrt{\frac{f_{\rho}}{K_{i}}} \frac{1}{f}\left(\left|\frac{f_{\rho \rho}}{2 f_{\rho}} \cdot \frac{\rho_{j} \rho_{\bar{k}}}{\sqrt{\rho_{j \bar{j}}} \sqrt{\rho_{k \bar{k}}}}\right|+\left|\frac{\rho_{j \bar{k}}}{\sqrt{\rho_{j \bar{j}}} \sqrt{\rho_{k \bar{k}}}}\right|\right)
$$

and so $\left|\left\langle\nabla_{Z_{i}^{j}} \Xi_{i}, \bar{Z}_{i}^{k}\right\rangle_{g_{i}(t)}\right| \rightarrow 0$ as $i \rightarrow \infty$ since by Lemma 5.2 we have $f_{\rho}=O(T-t)$. Finally, we have

$$
\begin{aligned}
\nabla_{\bar{Z}_{i}^{j}} \Xi_{i} & =\frac{1}{\sqrt{K_{i} g_{j \bar{j}}}} \frac{\partial}{\partial \bar{z}_{j}}\left(\frac{1}{\sqrt{K_{i} f_{\rho}} \rho_{\xi}} \frac{\partial}{\partial \xi}\right) \\
& =-\frac{1}{K_{i} f_{\rho \sqrt{g_{j \bar{j}}}}}\left(\frac{f_{\rho \rho}}{f_{\rho}}\right) \rho_{\bar{j}} \xi \frac{\partial}{\partial \xi},
\end{aligned}
$$




$$
\begin{gathered}
\left\langle\nabla_{\bar{Z}_{i}^{j}} \Xi_{i}, \bar{Z}_{i}^{k}\right\rangle_{g_{i}(t)}=-\frac{1}{\sqrt{K_{i} g_{j \bar{j}} g_{k \bar{k}}}}\left(\frac{f_{\rho \rho}}{f_{\rho}}\right) \rho_{\bar{j}} \rho_{\bar{k}}, \\
\left|\left\langle\nabla_{\bar{Z}_{i}^{j}} \Xi_{i}, \bar{Z}_{i}^{k}\right\rangle_{g_{i}(t)}\right| \leq \frac{1}{f \sqrt{K_{i}}}\left(\frac{f_{\rho \rho}}{f_{\rho}}\right) \cdot\left|\frac{\rho_{j} \rho_{k}}{\sqrt{\rho_{j \bar{j}} \rho_{k \bar{k}}}}\right| .
\end{gathered}
$$

Hence $\left|\left\langle\nabla_{\bar{Z}_{i}^{j}} \Xi_{i}, \bar{Z}_{i}^{k}\right\rangle_{g_{i}(t)}\right| \rightarrow 0$ as $i \rightarrow \infty$.

Since $\left\{Z_{\infty}^{j}, \Xi_{\infty}\right\}_{j=1}^{n}$ spans the whole $T_{\mathbb{C}} M_{\infty}$, the above calculations show that for any vector field $X$ on $\left(M_{\infty}, g_{\infty}(t)\right)$, one has $\left\langle\nabla_{X} \Xi_{\infty}, \bar{Z}_{\infty}^{j}\right\rangle_{g_{\infty}}=0$ for any $j=$ $1,2, \ldots, n$. Therefore, $\nabla_{X} \Re\left(\Xi_{\infty}\right), \nabla_{X} \Im\left(\Xi_{\infty}\right) \in E_{\infty}$. This shows whenever we have $\left.V_{x} \in E_{\infty}\right|_{x}, x \in M_{\infty}$, and let $V(s) \in T M$ be the parallel translation of $V_{x}$ along a curve $\gamma(s)$, then $V(s) \in E_{\infty}$. To see this, write $V(s)=V^{T}(s)+V^{\perp}(s)$ where $V^{T}(s) \in E_{\infty}$ and $V^{\perp}(s) \in E_{\infty}^{\perp}$ for any $s$. By the above calculation, we have $\nabla_{\gamma^{\prime}(s)} V^{T}(s) \in E_{\infty}$ for any $s$. Therefore,

$$
0=\nabla_{\gamma^{\prime}(s)} V(s)=\nabla_{\gamma^{\prime}(s)} V^{T}(s)+\nabla_{\gamma^{\prime}(s)} V^{\perp}(s) .
$$

Hence $\nabla_{\gamma^{\prime}(s)} V^{\perp}(s)$ also lies inside $E_{\infty}$. By the fact that $V^{\perp}(s) \perp E_{\infty}$, we have

$$
\frac{d}{d s}\left\|V^{\perp}(s)\right\|^{2}=2\left\langle\nabla_{\gamma^{\prime}(s)} V^{\perp}(s), V^{\perp}(s)\right\rangle=0 .
$$

It implies that $\left\|V^{\perp}(s)\right\| \equiv\left\|V^{\perp}(0)\right\|=0$ for any $s$. In other words, $V(s) \equiv V^{T}(s) \in$ $E_{\infty}$ for any $s$. Therefore, $E_{\infty}$ is invariant under parallel transport. By the de Rham decomposition theorem, our splitting lemma follows.

\section{Singularity ANALYSIS}

The splitting lemma in the previous section allows a dimension reduction for our singularity analysis. The ultimate goal of this section is to analyze the singularity formation of the Ricci flow on our projective bundles $M=\mathbb{P}\left(\mathcal{O}_{\Sigma} \oplus L\right)$ whose $\mathbb{P}^{1}$ fiber collapses near the singularity. We are going to prove that the Kähler-Ricci flow $(M, g(t))$ must be of Type I (see definition below) and the singularity model is $\mathbb{C}^{n} \times \mathbb{P}^{1}$, in a sense that one can choose a sequence $\left(x_{i}, t_{i}\right)$ in space-time in the high curvature region such that the universal cover of the Cheeger-Gromov limit of the rescaled dilated sequence is isometric to $\left(\mathbb{C}^{n} \times \mathbb{P}^{1},\|d \mathbf{z}\|^{2} \oplus \omega_{\mathrm{FS}}(t)\right)$. Here $\omega_{\mathrm{FS}}(t)$ is the shrinking Fubini-Study metric.

According to the blow-up rate of the Riemann curvature tensor, the singularity type of a Ricci flow solution which encounters finite-time singularity is classified as in [H3].

Definition 7.1. Let $(M, g(t))$ be a Ricci flow solution $\partial_{t} g(t)=-\operatorname{Ric}(g(t))$ on a closed manifold $M$ which becomes singular at a finite time $T$. We call the Ricci flow encounters

- Type I singularity if $\sup _{M \times[0, T)}(T-t)\|\mathrm{Rm}\|_{g(t)}<\infty$;

- Type II singularity if $\sup _{M \times[0, T)}(T-t)\|\mathrm{Rm}\|_{g(t)}=\infty$.

We would like to remark that although the Type I/II classification of finite-time singularity was proposed in the early 90s, surprisingly the first compact Type II solution was constructed by Gu-Zhu in GZ] only recently in 2007.

In order to understand the singularity formation, we need to bring curvatures into the topic. Therefore, we will compute and analyze the Riemann curvature tensor of our projective bundle $M$ which is equipped with momentum profile $f$. Recall that 
for Kähler manifolds, the Riemann curvature $(3,1)$-tensor can be computed using the formula

$$
R_{A \bar{B} C}^{D}=-\frac{\partial}{\partial \bar{z}^{B}} \Gamma_{A C}^{D}
$$

where $A, B, C, D=1, \ldots, n$ or $\xi$. The non-zero components of the Riemann curvature tensor are given below. For the ease of inspection of the norm $\|\mathrm{Rm}\|$ later on, we will split the components into five groups according to the number of $\xi$-indexes:

$$
\begin{aligned}
& R_{i \bar{j} k}^{l}=-(\log f)_{\rho \rho} \rho_{\bar{j}}\left(\rho_{i} \delta_{k l}+\rho_{k} \delta_{i l}\right)-(\log f)_{\rho}\left(\delta_{i j} \delta_{k l}+\delta_{j k} \delta_{i l}\right)-\left(\rho^{l \bar{p}} \rho_{i k \bar{p}}\right)_{\bar{j}}, \\
& R_{i \bar{\xi} k}^{l}=-(\log f)_{\rho \rho} \rho_{\bar{\xi}}\left(\rho_{i} \delta_{k l}+\rho_{k} \delta_{i l}\right), \\
& R_{i \bar{j} \xi}^{l}=-(\log f)_{\rho \rho} \rho_{\bar{j}} \rho_{\xi} \delta_{i l} \text {, } \\
& R_{i \bar{j} k}^{\xi}=-\frac{1}{\rho_{\xi}}\left(\log f_{\rho}-2 \log f\right)_{\rho \rho} \rho_{\bar{j}} \rho_{i} \rho_{k} \\
& -\frac{1}{\rho_{\xi}}\left(\log f_{\rho}-2 \log f\right)_{\rho}\left(\rho_{i \bar{j}} \rho_{k}+\rho_{k \bar{j}} \rho_{i}\right)+\frac{1}{\rho_{\xi}}\left(\rho^{l \bar{p}} \rho_{l} \rho_{i \bar{p} k}+\rho_{i k}\right)_{\bar{j}}, \\
& R_{\xi \bar{j} k}^{l}=-(\log f)_{\rho \rho} \rho_{\bar{j}} \rho_{\xi} \delta_{k l}, \\
& R_{i \bar{\xi} k}^{\xi}=-\left(\log f_{\rho}-2 \log f\right)_{\rho \rho} \frac{\rho_{\bar{\xi}}}{\rho_{\xi}} \rho_{i} \rho_{k}, \\
& R_{i \bar{\xi} \xi}^{l}=-(\log f)_{\rho \rho}\left|\rho_{\xi}\right|^{2} \delta_{i k}, \\
& R_{\xi \bar{\xi} k}^{l}=-(\log f)_{\rho \rho}\left|\rho_{\xi}\right|^{2} \delta_{k l}, \\
& R_{\xi \bar{j} \xi}^{l}=0 \text {, } \\
& R_{\xi \bar{j} k}^{\xi}=-\left(\log f_{\rho}-\log f\right)_{\rho \rho} \rho_{\bar{j}} \rho_{k}-\left(\log f_{\rho}-\log f\right)_{\rho} \rho_{k \bar{j}}, \\
& R_{l \bar{j} \xi}^{\xi}=-\left(\log f_{\rho}-\log f\right)_{\rho \rho} \rho_{\bar{j}} \rho_{l}-\left(\log f_{\rho}-\log f\right)_{\rho} \rho_{l \bar{j}}, \\
& R_{l \bar{\xi} \xi}^{\xi}=-\left(\log f_{\rho}-\log f\right)_{\rho \rho} \rho_{\bar{\xi}} \rho_{i}, \\
& R_{\xi \bar{\xi} \xi}^{l}=0 \text {, } \\
& R_{\xi \bar{\xi} k}^{\xi}=-\left(\log f_{\rho}-\log f\right)_{\rho \rho} \rho_{\bar{\xi}} \rho_{k}, \\
& R_{\xi \bar{j} \xi}^{\xi}=-\left(\log f_{\rho}\right)_{\rho \rho} \rho_{\bar{j}} \rho_{\xi}, \\
& R_{\xi \bar{\xi} \xi}^{\xi}=-\left(\log f_{\rho}\right)_{\rho \rho}\left|\rho_{\xi}\right|^{2} .
\end{aligned}
$$

Since the understanding of $\|\mathrm{Rm}\|$ is crucial in analyzing the singularity according to their type (I or II), we need an organized expression of $\|\mathrm{Rm}\|$ that is written in terms of our momentum profile $f$. Obviously, it would take loads of unnecessary work. However, in order to study the singularity model in our class of manifolds, it suffices to understand the asymptotics of $\|\mathrm{Rm}\|^{2}$ in terms of $f$ and its derivatives. 
Recall from Lemma 5.2 that $f=O(1), \frac{1}{f}=O(1), f_{\rho \rho} / f_{\rho}=O(1)$. Therefore we have the following asymptotics:

$$
\begin{aligned}
(\log f)_{\rho} & =\frac{f_{\rho}}{f}=O\left(f_{\rho}\right), \\
(\log f)_{\rho \rho} & =\frac{f_{\rho \rho}}{f}-\frac{f_{\rho}^{2}}{f^{2}}=O\left(f_{\rho}\right), \\
\left(\log f_{\rho}\right)_{\rho} & =\frac{f_{\rho \rho}}{f_{\rho}}=O(1) .
\end{aligned}
$$

The asymptotic of $\left(\log f_{\rho}\right)_{\rho \rho}$ is not yet known because it involves the third $\rho$ derivative of $f$, which we have not derived.

Also, the local expressions of $g$ and $g^{-1}$ have the following asymptotics:

$$
\begin{aligned}
& g_{i \bar{j}}=O(1), \\
& g_{i \bar{\xi}}=g_{\overline{i \xi}}=g_{\xi \bar{\xi}}=O\left(f_{\rho}\right), \\
& g^{i \bar{j}}=g^{i \bar{\xi}}=g^{\bar{i} \xi}=O(1), \\
& g^{\xi \bar{\xi}}=O\left(f_{\rho}^{-1}\right) .
\end{aligned}
$$

We claim that the norm $\|\mathrm{Rm}\|^{2}$ can be expressed in the following asymptotic form

\section{Lemma 7.2 .}

$$
\begin{aligned}
\|\mathrm{Rm}\|_{g(t)}^{2}= & f_{\rho}^{-2}\left(\log f_{\rho}\right)_{\rho \rho}^{2}+O\left(f_{\rho}^{-1}\left(\log f_{\rho}\right)_{\rho \rho}\right) \\
& +O\left(f_{\rho}^{-1}\left(\log f_{\rho}\right)_{\rho \rho}^{2}\right)+O\left(\left(\log f_{\rho}\right)_{\rho \rho}^{2}\right) \\
& +O\left(\left(\log f_{\rho}\right)_{\rho \rho}\right)+O(1) .
\end{aligned}
$$

Proof. A generic term in $\|\mathrm{Rm}\|^{2}$ can be expressed as

$$
g_{A \bar{B}} g^{C \bar{D}} g^{E \bar{F}} g^{G \bar{H}} R_{C \bar{F} G}^{A} \overline{R_{D \bar{E} H}^{B}}
$$

where $A, \ldots, H \in\{1, \ldots, n, \xi\}$. From Lemma 5.2. we know $f_{\rho}=O(T-t)$, and so $f_{\rho}^{-1}$ is a bad term as it diverges as $t \rightarrow T$. The only factor in (**) which can contribute to a $f_{\rho}^{-1}$ is $g^{\xi \bar{\xi}}$, and there are at most three $g^{\xi \bar{\xi}}$ 's in (**). We are going to check that

(1) whenever $f_{\rho}^{-1}$ appears in (**) exactly once, there must be at least one factor of $\left(\log f_{\rho}\right)_{\rho \rho}$ from the curvature components;

(2) whenever $f_{\rho}^{-2}$ appears in (**), there must be a $\left(\log f_{\rho}\right)_{\rho \rho}^{2}$ factor from the curvature components;

(3) it is impossible for $f_{\rho}^{-3}$ to appear in $* *$.

Combining these, it is not difficult to see $\|\mathrm{Rm}\|^{2}$ satisfies the asymptotic form (7.1).

We start by arguing (1). Suppose there is exactly one $f_{\rho}^{-1}$ factor in (**); we can assume WLOG that either $(C, D)=(\xi, \xi)$ or $(E, F)=(\xi, \xi)$. Suppose the former, we can check from the list of Riemann curvatures following Definition 7.1 that almost all $R_{\xi \bar{F} G}^{A}$ terms have either asymptotics $O\left(f_{\rho}\right)$ (which cancels out $f_{\rho}^{-1}$ ) or a $\left(\log f_{\rho}\right)_{\rho \rho}$ factor. There is only one exception: $R_{\xi \bar{j} k}^{\xi}$ which has an $O(1)$-term from $\left(\log f_{\rho}\right)_{\rho}$. However, if both of $R_{C \bar{F} G}^{A}$ and $R_{D \bar{E} H}^{B}$ are taken to be in this form, then ${ }^{* *}$ becomes

$$
g_{\xi \bar{\xi}} g^{\xi \bar{\xi}} g^{p \bar{j}} g^{k \bar{q}} R_{\xi \bar{j} k}^{\xi} \overline{R_{\xi \bar{p} q}^{\xi}}
$$


where the $g_{\xi \bar{\xi}}=O\left(f_{\rho}\right)$ cancels out the undesirable $f_{\rho}^{-1}$ factor, and we end up with no $f_{\rho}^{-1}$ at all. A similar argument applies to the case $(E, F)=(\xi, \xi)$, and (1) is proved.

For (2), since $g^{\xi \bar{\xi}}$ is the only possible contribution to $f_{\rho}^{-1}$, at least two of $C, F, G$ (and their corresponding two of $D, E, G$ ) must be $\xi$. Check again the list of Riemann curvature components following Definition 7.1; we see all the terms with two lower $\xi$-indexes must either be of $O\left(f_{\rho}\right)$-type or have a $\left(\log f_{\rho}\right)_{\rho \rho}$ factor. It proves $(2)$.

For (3), the only possible case for $f_{\rho}^{-3}$ to appear is that all of $(C, D),(E, F)$ and $(G, H)$ are $(\xi, \xi)$. The only possible choice for the curvature components are $R_{\xi \bar{\xi} \xi}^{l}$ and $R_{\xi \bar{\xi} \xi}^{\xi}$. However, the former is 0 . For the latter case, all indexes will be $\xi$ and (**) becomes

$$
g_{\xi \bar{\xi}} g^{\xi \bar{\xi}} g^{\xi \bar{\xi}} g^{\xi \bar{\xi}} R_{\xi \bar{\xi} \xi}^{\xi} \overline{R_{\xi \bar{\xi} \xi}^{\xi}}
$$

which can be computed easily as $f_{\rho}^{-2}\left(\log f_{\rho}\right)_{\rho \rho}^{2}$.

Finally, we remark that $g_{\xi \bar{\xi}} g^{\xi \bar{\xi}} g^{\xi \bar{\xi}} g^{\xi \bar{\xi}} R_{\xi \bar{\xi} \xi}^{\xi} \overline{R_{\xi \bar{\xi} \xi}^{\xi}}$ is the only term where $f_{\rho}^{-2}\left(\log f_{\rho}\right)_{\rho \rho}^{2}$ appears, thanks to the fact that $R_{\xi \bar{\xi} \xi}^{i}=0$. As a result, the leading term of (7.1) is $f_{\rho}^{-2}\left(\log f_{\rho}\right)_{\rho \rho}^{2}$ with coefficient 1 which can be easily verified by computing $g_{\xi \bar{\xi}} g^{\xi \bar{\xi}} g^{\xi \bar{\xi}} g^{\xi \bar{\xi}} R_{\xi \bar{\xi} \xi}^{\xi} \overline{R_{\xi \bar{\xi} \xi}^{\xi}}$.

Having understood the asymptotics of $\|\mathrm{Rm}\|^{2}$, we are in a position to study the singularity models. Let's first consider the Type I case:

Theorem 7.3. Let $M=\mathbb{P}\left(\mathcal{O}_{\Sigma} \oplus L\right)$ be the projective bundle with the triple $\left(\Sigma, L,\left[\omega_{0}\right]\right)$ satisfying the conditions listed in the subsection on main results. Let $\left(M, \omega_{t}\right)$ be the Kähler-Ricci flow $\partial_{t} \omega_{t}=-\operatorname{Ric}\left(\omega_{t}\right), t \in[0, T)$, with initial Kähler class $\left[\omega_{0}\right]$. Suppose the flow encounters Type I singularity; then choose $\left(x_{i}, t_{i}\right)$ in space-time such that $K_{i}:=\left\|\operatorname{Rm}\left(x_{i}, t_{i}\right)\right\|_{g\left(t_{i}\right)}=\max _{M}\|\mathrm{Rm}\|_{g\left(t_{i}\right)}$ and $t_{i} \rightarrow T$. Consider the rescaled dilated sequence of metrics $g_{i}(t):=K_{i} g\left(t_{i}+K_{i}^{-1} t\right), t \in\left[-t_{i} K_{i},(T-\right.$ $\left.\left.t_{i}\right) K_{i}\right)$. Then the pointed sequence $\left(M, g_{i}(t), x_{i}\right)$ converges, after passing to a subsequence, smoothly in the pointed Cheeger-Gromov sense to an ancient $\kappa$-solution $\left(M_{\infty}, g_{\infty}(t), x_{\infty}\right)$, whose universal cover splits isometrically as

$$
\left(\mathbb{C}^{n} \times \mathbb{P}^{1},\|d \mathbf{z}\|^{2} \oplus \omega_{F S}(t)\right),
$$

where $\|d \mathbf{z}\|^{2}$ is the Euclidean metric and $\omega_{F S}(t)$ denotes the shrinking Fubini-Study metric.

Proof. Suppose $C=C(n)$ is a constant depending only on $n$ such that $|R| \leq$ $C(n)\|\mathrm{Rm}\|$. Since the blow-up factor $K_{i}$ is defined by $K_{i}=\max _{M}\|\mathrm{Rm}\|_{g\left(t_{i}\right)}=$ $\left\|\operatorname{Rm}\left(x_{i}\right)\right\|_{g\left(t_{i}\right)}$, the scalar curvature at time $t_{i}$ satisfies $\left|R\left(g\left(t_{i}\right)\right)\right| \leq C K_{i}$ on $M$.

One can compute the scalar curvature explicitly:

$$
\begin{aligned}
R_{g(t)} & =\operatorname{Tr}_{\omega_{t}} \operatorname{Ric}\left(\omega_{t}\right) \\
& =\frac{n\left(\nu-F_{\rho}\right)}{f}-\frac{F_{\rho \rho}}{f_{\rho}},
\end{aligned}
$$

where $F=\log f_{\rho}+n \log f$. Hence,

$$
R_{g(t)}=-\frac{1}{f_{\rho}}\left(\log f_{\rho}\right)_{\rho \rho}+O(1) .
$$


Therefore, for any $\rho \in[-\infty, \infty]$ at $t_{i}$, we have

$$
\begin{aligned}
\left|-\frac{1}{f_{\rho}}\left(\log f_{\rho}\right)_{\rho \rho}+O(1)\right| & \leq C K_{i}, \\
\left|\frac{-1}{K_{i} f_{\rho}}\left(\log f_{\rho}\right)_{\rho \rho}+O\left(K_{i}^{-1}\right)\right| & \leq C .
\end{aligned}
$$

Recall that $K_{i} \rightarrow \infty$. Letting $i \rightarrow \infty$ yields

$$
\limsup _{i \rightarrow \infty}\left|K_{i}^{-1} f_{\rho}^{-1}\left(\log f_{\rho}\right)_{\rho \rho}\right|_{\left(\rho, t_{i}\right)} \leq C .
$$

By considering the asymptotic expression of $\|\mathrm{Rm}\|^{2}$ given by (7.1), we have for any $\rho \in[-\infty, \infty]$ at time $t_{i}$,

$$
\begin{aligned}
1 \geq K_{i}^{-2}\|\mathrm{Rm}\|_{g\left(t_{i}\right)}^{2}= & \left(K_{i} f_{\rho}\right)^{-2}\left(\log f_{\rho}\right)_{\rho \rho}^{2}+O\left(K_{i}^{-2} f_{\rho}^{-1}\left(\log f_{\rho}\right)_{\rho \rho}^{2}\right) \\
& +O\left(K_{i}^{-2} f_{\rho}^{-1}\left(\log f_{\rho}\right)_{\rho \rho}\right)+O\left(K_{i}^{-2}\left(\log f_{\rho}\right)_{\rho \rho}^{2}\right) \\
& +O\left(K_{i}^{-2}\left(\log f_{\rho}\right)_{\rho \rho}\right)+O\left(K_{i}^{-2}\right),
\end{aligned}
$$

where equality is achieved at $x_{i}$.

Letting $i \rightarrow \infty$ and using (7.2) and the fact that $f_{\rho}=O(T-t)$ from Lemma 5.2, we can deduce:

$$
\begin{aligned}
\limsup _{i \rightarrow \infty}\left(K_{i} f_{\rho}\right)^{-2}\left(\log f_{\rho}\right)_{\rho \rho}^{2} \leq 1, \quad \rho \in[-\infty, \infty], \quad t=t_{i}, \\
\left.\lim _{i \rightarrow \infty}\left(K_{i} f_{\rho}\right)^{-2}\left(\log f_{\rho}\right)_{\rho \rho}^{2}\right|_{\left(x_{i}, t_{i}\right)}=1 .
\end{aligned}
$$

Recall that $g_{i}(t)=K_{i} g\left(t_{i}+K_{i}^{-1} t\right)$; we then have

$$
R_{g_{i}(t)}=-\frac{1}{K_{i} f_{\rho}}\left(\log f_{\rho}\right)_{\rho \rho}+\left.O\left(K_{i}^{-1}\right)\right|_{t_{i}+K_{i}^{-1} t} .
$$

Letting $i \rightarrow \infty$, we have

$$
R_{g_{\infty}(t)}=-\left.\lim _{i \rightarrow \infty} \frac{1}{K_{i} f_{\rho}}\left(\log f_{\rho}\right)_{\rho \rho}\right|_{t_{i}+K_{i}^{-1} t} .
$$

By strong maximum principle, the scalar curvature of every ancient solution must be either identically zero or everywhere positive. In our case, (7.3) and (7.4) together imply $R_{g_{\infty}(0)}=1$ and hence $R_{g_{\infty}(t)}>0$ on $M \times(-\infty, 0]$. By our splitting lemma, Lemma 6.4, we know that the limit manifold $M_{\infty}$ splits isometrically as a product $N_{1}^{n} \times N_{2}^{1}$, such that $T N_{1}^{n}=\operatorname{span}_{\mathbb{R}}\left\{\Re\left(Z_{\infty}^{j}\right), \Im\left(Z_{\infty}^{j}\right)\right\}_{j=1}^{n}$ and $T N_{2}^{1}=\operatorname{span}_{\mathbb{R}}\left\{\Re\left(\Xi_{\infty}\right), \Im\left(\Xi_{\infty}\right)\right\}$. As a result, the curvature tensors also split as $\mathrm{Rm}_{M_{\infty}}=\mathrm{Rm}_{N_{1}^{n}} \oplus \mathrm{Ric}_{N_{2}^{1}}$. Next, we would like to compute the curvatures of each factor. Again, for simplicity we denote $Z_{g_{i}(t)}^{j}$ by $Z_{i}^{j}$ and $\Xi_{g_{i}(t)}$ by $\Xi_{i}$ :

$$
\begin{aligned}
& \left|\operatorname{Rm}_{g_{i}(t)}\left(Z_{i}^{j}, \bar{Z}_{i}^{k}, Z_{i}^{l}, \bar{Z}_{i}^{p}\right)\right| \\
& =\left|K_{i}\left\langle\operatorname{Rm}\left(Z_{i}^{j}, \bar{Z}_{i}^{k}\right) Z_{i}^{l}, \bar{Z}_{i}^{p}\right\rangle_{g\left(t_{i}+K_{i}^{-1} t\right)}\right| \\
& \leq\left|K_{i}\left(\frac{1}{\sqrt{K_{i}}}\right)^{4} \frac{1}{\sqrt{f \rho_{j \bar{j}}}} \frac{1}{\sqrt{f \rho_{k \bar{k}}}} \frac{1}{\sqrt{f \rho_{l \bar{l}}}} \frac{1}{\sqrt{f \rho_{p \bar{p}}}} R_{j \bar{k} l \bar{p}}\right| \\
& =\frac{1}{K_{i}} O(1) \rightarrow 0 \quad \text { as } i \rightarrow \infty .
\end{aligned}
$$


Hence $\operatorname{Rm}_{N^{n}}=0$. Similarly, we have

$$
\begin{aligned}
\operatorname{Ric}_{g_{i}(t)}\left(\Xi_{i}, \bar{\Xi}_{i}\right) & =\frac{1}{\sqrt{K_{i} f_{\rho}}} \frac{1}{\sqrt{K_{i} f_{\rho}}} \frac{1}{\left|\rho_{\xi}\right|^{2}}\left(-\left(n \log f+\log f_{\rho}\right)_{\rho \rho}\left|\rho_{\xi}\right|^{2}\right) \\
& =-\frac{1}{K_{i} f_{\rho}}\left(\log f_{\rho}\right)_{\rho \rho}+O\left(K_{i}^{-1}\right) .
\end{aligned}
$$

By (7.4) and positivity of $R_{g_{\infty}(t)}$, we know that $\operatorname{Ric}_{g_{\infty}(t)}\left(\Xi_{\infty}, \bar{\Xi}_{\infty}\right)>0$.

Since the Kähler-Ricci flow $g(t)$ is of Type I, the ancient solution obtained by the blow-up sequence is also of Type I, i.e. $\sup _{M \times(-\infty, 0]}|t|\|\mathrm{Rm}\|_{g_{\infty}(t)}<\infty$, and is $\kappa$-non-collapsed. The limit solution splits as a product $\left(N_{1}^{n}, h_{1}(t)\right) \times\left(N_{2}^{1}, h_{2}(t)\right)$ where we know $N_{1}^{n}$ is flat and $N_{2}^{1}$ has positive curvature. According to Hamilton's classification of ancient $\kappa$-solution [H3] (see also [CLN]), $\left(N_{2}^{1}, h_{2}(t)\right)$ must be the shrinking round 2 -sphere.

To conclude, if the Kähler-Ricci flow $(M, g(t))$ is of Type I, then the universal cover of the limit solution $\left(M_{\infty}, g_{\infty}(t)\right)$ of the rescaled dilated sequence $g_{i}(t)$ is isometric to

$$
\left(\mathbb{C}^{n} \times \mathbb{P}^{1},\|d \mathbf{z}\|^{2} \oplus \omega_{\mathrm{FS}}(t)\right) .
$$

Next, we will rule out the possibility of Type II singularity on $(M, g(t))$. We will show that by a standard point-picking argument for Type II singularity, one can form a rescaled dilated sequence of metrics which converges, after passing to a subsequence, to a product of the cigar soliton and a flat factor. By Perelman's local non-collapsing result, such a limit model is not possible. Let's state this result and give its proof.

Theorem 7.4. Let $M=\mathbb{P}\left(\mathcal{O}_{\Sigma} \oplus L\right)$ be the projective bundle with the triple $\left(\Sigma, L,\left[\omega_{0}\right]\right)$ satisfying the conditions listed in the subsection on main results. Let $\left(M, \omega_{t}\right)$ be the Kähler-Ricci flow $\partial_{t} \omega_{t}=-\operatorname{Ric}\left(\omega_{t}\right), t \in[0, T)$, with initial Kähler class $\left[\omega_{0}\right]$. Then $(M, g(t))$ must be of Type I, i.e. Type II singularity is not possible.

Proof. First take an increasing sequence $T_{i} \rightarrow T$. Let $\left(x_{i}, t_{i}\right) \in M \times\left[0, T_{i}\right]$ be such that

$$
\begin{aligned}
\left(T_{i}-t_{i}\right)\|\operatorname{Rm}\|\left(x_{i}, t_{i}\right) & =\max _{M \times\left[0, T_{i}\right]}\left(T_{i}-t\right)\|\operatorname{Rm}\|_{g(t)} \\
& =\max _{M \times\left[-K_{i} t_{i}, K_{i}\left(T_{i}-t_{i}\right)\right]}\left(T_{i}-\left(t_{i}+K_{i}^{-1} t\right)\right)\|\mathrm{Rm}\|_{g\left(t_{i}+K_{i}^{-1} t\right)} .
\end{aligned}
$$

We denote $K_{i}=\|\operatorname{Rm}\|\left(\rho_{i}, t_{i}\right)$; then $K_{i}\left(T_{i}-t_{i}\right) \rightarrow \infty$ by the Type II condition.

As in the Type I case, we let $C=C(n)$ be a constant depending only on $n$ such that $\left|R_{g(t)}\right| \leq C\|\mathrm{Rm}\|_{g(t)}$. Recall that scalar curvature has the following asymptotic expression:

$$
R_{g(t)}=-\frac{1}{f_{\rho}}\left(\log f_{\rho}\right)_{\rho \rho}+O(1)
$$

Hence for any $\rho \in[-\infty, \infty], t \in\left[0, T_{i}\right]$, we have

$$
\begin{aligned}
\left|-\frac{1}{f_{\rho}}\left(\log f_{\rho}\right)_{\rho \rho}+O(1)\right| & \leq \frac{C\left(T_{i}-t_{i}\right) K_{i}}{T_{i}-\left(t_{i}+K_{i}^{-1} t\right)}, \\
\left|\frac{-1}{K_{i} f_{\rho}}\left(\log f_{\rho}\right)_{\rho \rho}+O\left(K_{i}^{-1}\right)\right| & \leq \frac{C\left(T_{i}-t_{i}\right)}{T_{i}-\left(t_{i}+K_{i}^{-1} t\right)},
\end{aligned}
$$


where we evaluate the left-hand side at $t_{i}+K_{i}^{-1} t$. Letting $i \rightarrow \infty$, and using the fact that $\frac{\left(T_{i}-t_{i}\right)-K_{i}^{-1} t}{T_{i}-t_{i}}=1-\frac{t}{K_{i}\left(T_{i}-t_{i}\right)} \rightarrow 1$, one can show

$$
\limsup _{i \rightarrow \infty}\left|\left(K_{i} f_{\rho}\right)^{-1}\left(\log f_{\rho}\right)_{\rho \rho}\right|_{\left(x, t_{i}+K_{i}^{-1} t\right)} \leq 1 \quad \text { for any }(x, t) .
$$

At $\left(x_{i}, t_{i}\right)$ we have $\left(T_{i}-t_{i}\right)^{2}\|\mathrm{Rm}\|^{2}\left(x_{i}, t_{i}\right)=\left(T_{i}-t_{i}\right)^{2} K_{i}^{2}$. Consider the asymptotic expression of $\|\mathrm{Rm}\|^{2}$ as in the Type I case; one can then show

$$
\left.\lim _{i \rightarrow \infty} \frac{1}{K_{i}^{2} f_{\rho}^{2}}\left(\log f_{\rho}\right)_{\rho \rho}^{2}\right|_{\left(x_{i}, t_{i}\right)}=1 .
$$

As $K_{i} \rightarrow \infty$, our splitting lemma, Lemma 6.4 also implies that the limit solution $\left(M_{\infty}, g_{\infty}(t)\right)$ splits isometrically as a product $\left(N_{1}^{n} \times N_{2}^{1}, h_{1}(t) \oplus h_{2}(t)\right)$. As in the Type I case, $\mathrm{Rm}_{N_{1}^{n}}$ and $\mathrm{Ric}_{N_{2}^{1}}$ can be found by (7.5) and (7.6):

$$
\begin{aligned}
\operatorname{Rm}_{g_{i}(t)}\left(Z_{i}^{j}, \bar{Z}_{i}^{k}, Z_{i}^{l}, \bar{Z}_{i}^{p}\right) & =\frac{1}{K_{i}} O(1), \\
\operatorname{Ric}_{g_{i}(t)}\left(\Xi_{i}, \bar{\Xi}_{i}\right) & =-\frac{1}{K_{i} f_{\rho}}\left(\log f_{\rho}\right)_{\rho \rho}+O\left(K_{i}^{-1}\right) .
\end{aligned}
$$

Letting $i \rightarrow \infty$, we have $\operatorname{Rm}_{N_{1}^{n}}\left(h_{1}(t)\right)=0$ and

$$
\begin{aligned}
& 1 \geq \operatorname{Ric}_{N_{2}^{1}}\left(h_{2}(t)\right)>0 \\
& \text { from (7.7), } \\
& \operatorname{Ric}_{N_{2}^{1}}\left(x_{\infty}, h_{2}(0)\right)=h_{2}(0) \\
& \text { from (7.8). }
\end{aligned}
$$

$\left(M_{\infty}, g_{\infty}(t)\right)$ is an eternal solution to the Kähler-Ricci flow since we have $\left(T_{i}-\right.$ $\left.t_{i}\right) K_{i} \rightarrow \infty$. By our splitting lemma, so does $\left(N_{2}^{1}, h_{2}(t)\right)$. From (7.9), the spacetime maximum of the scalar curvature of $\left(N_{2}^{1}, h_{2}(t)\right)$ is achieved at $\left(x_{\infty}, 0\right)$. Hence by Hamilton's classification of eternal solutions (see the Main Theorem of [H2]), $\left(N_{2}^{1}, h_{2}(t)\right)$ is a steady gradient soliton. In the case of $\operatorname{dim}_{\mathbb{R}}=2$, it must be the cigar soliton (see Section 26.3 of [H3]). However, by Perelman's local non-collapsing P1, the Cheeger-Gromov limit $\left(M_{\infty}, g(t)\right)$ must be $\kappa$-non-collapsed at all scales, and so the product of cigar soliton and a flat space is not a possible singularity model. It leads to a contradiction and hence completes our proof.

Remark 7.5. Throughout this paper we have focused on Case 1 and Case 2(i) following Theorem 4.1. We would like to point out as a final remark that for Case 2(iii) we expect one could mimic Section 5.2 in SW1 and also [SW2, SW3 to show the contraction of $\Sigma_{0}$ near the singular time. For singularity models obtained by rescaling analysis in Case 2(iii), it is conjectured in [FIK] that for $\left(\Sigma, \omega_{\Sigma}\right)=$ $\left(\mathbb{P}^{n}, \omega_{\mathrm{FS}}\right)$ the singularity should be modelled on Kähler-Ricci solitons on $\mathcal{O}(-k)$ bundles over $\mathbb{P}^{n}$.

\section{ACKNOWLEDGMENTS}

The author would like to express his heartfelt gratitude to his advisor, Professor Richard Schoen, for all his continuing support and many productive discussions. The author would also like to thank Yanir Rubinstein for arousing his interest in this topic and for many helpful ideas, and also Ziyu Zhang for informing him of some algebraic aspects related to this study. 


\section{REFERENCES}

[ACGT] Vestislav Apostolov, David M. J. Calderbank, Paul Gauduchon, and Christina W. Tønnesen-Friedman, Extremal Kähler metrics on ruled manifolds and stability, Astérisque (2008), no. 322, 93-150, Géométrie différentielle, physique mathématique, mathématiques et société. II.

[A] T. Aubin, Equations du type Monge-Ampére sur les Variétés Kählériennes, Compactes, C. R. Acad. Sci. Paris (1976), no. 283. MR0433520 (55:6496)

[BS] Simon Brendle and Richard Schoen, Manifolds with 1/4-pinched curvature are space forms, J. Amer. Math. Soc. 22 (2009), no. 1, 287-307. MR2449060 (2010a:53045)

[C] Eugenio Calabi, Extremal Kähler metrics, Seminar on Diff. Geom. Ann. of Math. Stud. 102 (1982), 259-290. MR645743 (83i:53088)

[Cao1] Huai Dong Cao, Deformation of Kähler metrics to Kähler-Einstein metrics on compact Kähler manifolds, Invent. Math. 81 (1985), no. 2, 359-372. MR799272 (87d:58051)

[Cao2] Huai-Dong Cao, Existence of gradient Kähler-Ricci solitons, Elliptic and parabolic methods in geometry (Minneapolis, MN, 1994), A K Peters, Wellesley, MA, 1996, pp. 1-16. MR.1417944 (98a:53058)

[CCG] Bennett Chow, Sun-Chin Chu, David Glickenstein, Christine Guenther, James Isenberg, Tom Ivey, Dan Knopf, Peng Lu, Feng Luo, and Lei Ni, The Ricci flow: techniques and applications. Part I, Mathematical Surveys and Monographs, vol. 135, American Mathematical Society, Providence, RI, 2007, Geometric aspects. MR2302600 (2008f:53088)

[CLN] Bennett Chow, Peng Lu, and Lei Ni, Hamilton's Ricci flow, Graduate Studies in Mathematics, vol. 77, American Mathematical Society, Providence, RI, 2006. MR 2274812 (2008a:53068)

[CY] Shiu Yuen Cheng and Shing Tung Yau, On the existence of a complete Kähler metric on noncompact complex manifolds and the regularity of Fefferman's equation, Comm. Pure Appl. Math. 33 (1980), no. 4, 507-544. MR575736 (82f:53074)

[CZ] Huai-Dong Cao and Xi-Ping Zhu, Hamilton-Perelman's Proof of the Poincaré Conjecture and the Geometrization Conjecture, Asian J. Math. 10 (2006), no. 2, 165-492. MR.2233789 (2008d:53090)

[DW] Andrew S. Dancer and McKenzie Y. Wang, On Ricci solitons of cohomogeneity one, Ann. Global Anal. Geom. 39 (2011), no. 3, 259-292. MR2769300

[FIK] M. Feldman, T. Ilmanen, and D. Knopf, Rotationally symmetric shrinking and expanding gradient Kähler-Ricci solitons, Journal of Differential Geometry 65 (2003), no. 2, 169209. MR2058261 (2005e:53102)

[GH] Phillip Griffiths and Joseph Harris, Principles of algebraic geometry, Wiley Classics Library, John Wiley \& Sons Inc., New York, 1994, Reprint of the 1978 original. MR 1288523 (95d:14001)

[GZ] Hui-Ling Gu and Xi-Ping Zhu, The existence of type II singularities for the Ricci flow on $S^{n+1}$, Comm. Anal. Geom., 16 (2008), no. 3, 467-494. MR2429966 (2009k:53169)

[H1] Richard S. Hamilton, Three-manifolds with positive Ricci curvature, J. Differential Geom. 17 (1982), no. 2, 255-306. MR664497(84a:53050)

[H2] _ Eternal solutions to the Ricci flow, J. Differential Geom. 38 (1993), no. 1, 1-11. MR.1231700 (94g:58043)

[H3] , The formation of singularities in the Ricci flow, Surveys in Diff. Geom., 2 (1995), 7-136. MR1375255 (97e:53075)

[HS] Andrew D. Hwang and Michael A. Singer, A momentum construction for circle-invariant Kähler metrics, Trans. Amer. Math. Soc. 354 (2002), no. 6, 2285-2325 (electronic). MR:1885653 (2002m:53057)

[KL] Bruce Kleiner and John Lott, Notes on Perelman's papers, Geom. Topol. 12 (2008), no. 5, 2587-2855. MR2460872 (2010h:53098)

[Koi] Norihito Koiso, On rotationally symmetric Hamilton's equation for Kähler-Einstein metrics, Recent topics in differential and analytic geometry, Adv. Stud. Pure Math., vol. 18, Academic Press, Boston, MA, 1990, pp. 327-337. MR.1145263 (93d:53057)

[Li] Chi Li, On rotationally symmetric Kahler-Ricci solitons, Apr 2010, arxiv:1004.4049

[MT] John W. Morgan and Gang Tian, Ricci flow and the Poincare Conjecture, Clay Math. Monographs, 3 (2007), American Math. Soc. MR2334563 (2008d:57020) 
[Mum] David Mumford, Abelian varieties, Tata Institute of Fundamental Research Studies in Mathematics, No. 5, 1970. MR0282985 (44:219)

[P1] Grisha Perelman, The entropy formula for the Ricci flow and its geometric applications, November 2002, arxiv:math/0211159

[P2] _ Ricci flow with surgery on three-manifolds, March 2003, arixv:math/0307245

[P3] Finite extinction time for the solutions to the Ricci flow on certain threemanifolds, July 2003, arxiv:math/0307245

[Shi] Wan-Xiong Shi, Ricci deformation of the metric on complete noncompact Riemannian manifolds, J. Differential Geom. 30 (1989), no. 2, 303-394. MR.1010165 (90f:53080)

[SSW] Jian Song, Gábor Székelyhidi, and Ben Weinkove, The Kähler-Ricci flow on projective bundles, July 2011, arxiv:1107.2144

[ST1] Jian Song and Gang Tian, The Kähler-Ricci flow on surfaces of positive Kodaira dimension, Invent. Math., 170 (2007), no. 3, 609-653. MR2357504 (2008m:32044)

[ST2] Natasa Sesum and Gang Tian, Bounding scalar curvature and diameter along the Kähler Ricci flow (after Perelman), J. Inst. Math. Jussieu 7 (2008), no. 3, 575-587. MR2427424 (2009c:53092)

[ST3] Jian Song and Gang Tian, The Kähler-Ricci flow through singularities, Sep. 2009, arxiv:0909.4898

[SW1] Jian Song and Ben Weinkove, The Kähler-Ricci flow on Hirzebruch surfaces, to appear in J. Reine Angew. Math, arxiv:0903.1900

[SW2] - Contracting exceptional divisors by the Kähler-Ricci flow, Mar. 2010, arxiv:1003.0718

[SW3] Contracting exceptional divisors by the Kähler-Ricci flow II, Feb. 2011, arxiv:1102.1759

[T] _ New results and problems on Kähler-Ricci flow, Astérisque, Géométrie différentielle, physique mathématique, mathématiques et société. II (2008), no. 322, 7192.

[TZ] Gang Tian and Zhou Zhang, On the Kähler-Ricci flow on projective manifolds of general type, Chinese Ann. Math. Ser. B 27 (2006), no. 2, 179-192. MR2243679 (2007c:32029)

[Yg] Bo Yang, A characterization of Koiso's type solitons, Feb 2008, arxiv:0802.0300

[Y] S. T. Yau, On the Ricci curvature of a compact Kähler manifold and the complex MongeAmpère equation, I, Comm. Pure Appl. Math (1978), no. 31, 339-411. MR480350 (81d:53045)

Department of Mathematics, Stanford University, Stanford, California 94305

E-mail address: thfong@math.stanford.edu

Current address: Department of Mathematics, Brown University, 151 Thayer Street, Box 1917, Providence, Rhode Island 02912 